ApJ Accepted 2015.July.22

\title{
SOFIA Infrared Spectrophotometry of Comet C/2012 K1 (Pan-STARRS)
}

\author{
Charles E. Woodward ${ }^{1}$, Michael S. P. Kelley ${ }^{2}$, David E. Harker ${ }^{3}$, Erin L. Ryan², \\ Diane H. Wooden ${ }^{4}$, Michael L. Sitko ${ }^{5}$, Ray W. Russell ${ }^{6}$, \\ William T. Reach ${ }^{7}$, Imke de Pater $^{8}$, Ludmilla Kolokolova ${ }^{2}$, Robert D. Gehrz ${ }^{9}$
}

\begin{abstract}
We present pre-perihelion infrared 8 to $31 \mu \mathrm{m}$ spectrophotometric and imaging observations of comet $\mathrm{C} / 2012 \mathrm{~K} 1$ (Pan-STARRS), a dynamically new Oort Cloud comet, conducted with NASA's Stratospheric Observatory for Infrared Astronomy (SOFIA) facility (+FORCAST) in 2014 June. As a "new" comet (first inner solar system passage), the coma grain population may be extremely pristine, unencumbered by a rime and insufficiently irradiated by the Sun to carbonize its surface organics. The comet exhibited a weak $10 \mu \mathrm{m}$ silicate feature $\simeq 1.18 \pm 0.03$ above the underlying best-fit $215.32 \pm 0.95 \mathrm{~K}$ continuum blackbody. Thermal modeling of the observed spectral energy distribution indicates that the coma grains are fractally solid with a porosity factor $D=3$ and the peak in the grain size distribution, $a_{\text {peak }}=0.6 \mu \mathrm{m}$,
\end{abstract}

\footnotetext{
${ }^{1}$ Minnesota Institute for Astrophysics, University of Minnesota, 116 Church St, SE Minneapolis, MN 55455, USA; chickw024@gmail.com

${ }^{2}$ University of Maryland, Department of Astronomy, College Park, MD 20742-2421, USA

${ }^{3}$ University of California, San Diego, Center for Astrophysics \& Space Sciences, 9500 Gilman Dr. Dept 0424, La Jolla, CA 92093-0424, USA

${ }^{4}$ NASA Ames Research Center, MS 245-3, Moffett Field, CA 94035-0001, USA

${ }^{5}$ Department of Physics, University of Cincinnati, Cincinnati, OH 45221, USA

${ }^{6}$ The Aerospace Corporation, Los Angeles, CA 90009, USA

${ }^{7}$ USRA-SOFIA Science Center, NASA Ames Research Center, Moffett Field, CA 94035, USA

${ }^{8}$ Astronomy Department, 601 Campbell Hall, University of California, Berkeley, CA 94720, USA

${ }^{9}$ Minnesota Institute for Astrophysics, University of Minnesota, 116 Church St, SE Minneapolis, MN $55455, \mathrm{USA}$
} 
large. The sub-micron coma grains are dominated by amorphous carbon, with a silicate-to-carbon ratio of $0.80_{-0.20}^{+0.25}$. The silicate crystalline mass fraction is $0.20_{-0.10}^{+0.30}$, similar to with other dynamically new comets exhibiting weak $10 \mu \mathrm{m}$ silicate features. The bolometric dust albedo of the coma dust is $0.14 \pm 0.01$ at a phase angle of $34.76^{\circ}$, and the average dust production rate, corrected to zero phase, at the epoch of our observations was $A f \rho \simeq 5340 \mathrm{~cm}$.

Subject headings: comets: general - comets: individual (C/2012 K1 PanSTARRS) - ISM: dust

\section{INTRODUCTION}

Solar System formation was an engine that simultaneously preserves and transforms interstellar medium (ISM) ices, organics, and dust grains into cometesimals, planetesimals and, ultimately, planets. Observing and modeling the properties of small, primitive bodies in the solar system whose origins lie beyond the water frost line $(>5 \mathrm{AU})$ provides critical insight into the formation of Solar System solids and establishes observation constraints for planetary system formation invoking migration - the 'Grand Tack' epoch (Walsh et al. 2011), followed by the 'Nice Model' events (Levison 2009; Gomes et al. 2005). The characteristics of comet dust can provide evidence to validate the new, emerging picture of small body populations - including comet families - resulting from planetary migration in the early Solar System.

Inside cometary nuclei, the bulk of the dust likely has been preserved since formation of the nucleus. Comet grains (and ices) also trace the pre-accretion history of comet materials extant in the outer disk. Comet dust composition can be studied via Stardust samples, selected collections of Interplanetary Dust Particles (IDPs), and in situ analysis in comet flyby and/or rendezvous missions. Dust species that are best explained as products of aqueous alteration (e.g., magnitite, cubanite, possibly pentlandite) are rare (Stodolna et al. 2012; Berger et al. 2011; Zolensky et al. 2008) and corresponding altered silicates (e.g., phyllosilicates, smectite) are missing suggesting that aqueous alteration in cometary nuclei is limited, is not well represented in the Stardust samples, or that these minerals have exogenous origins (Brownlee 2014). Thus, the bulk of comet grain properties including dust size, porosity, and composition relate to grain formation, radial mixing, and particle agglomeration in the proto-solar disk (for an extensive review see Brownlee 2014). However, opportunities to study actual samples of cometary dust are rare, motivating the need for telescopic remote sensing observations of dust whenever apparitions are accessible from terrestrial observatories. 
In this paper we report our pre-perihelion ( $\mathrm{TP}=2014$ Aug 27.65 UT) infrared 8 to 31 micron spectrophotometric observations of comet C/2012 K1 (Pan-STARRS), a dynamically new (see Oort 1950, for a definition based on orbital elements) Oort Cloud comet $-\left(1 / a_{\text {org }}\right)=$ $42.9 \times 10^{-6} \mathrm{AU}^{-1}$ (Williams 2015) - conducted with NASA's Stratospheric Observatory for Infrared Astronomy (SOFIA) facility during a series of four flights over the period from 2014 June 04 to 13 UT. Contemporaneous optical imaging observations are also presented.

\section{OBSERVATIONS}

\subsection{Ground-based Optical Imaging}

Comet C/2012 K1 (Pan-STARRS) was observed on 2012 June 01.22 UT and again on June 04.24 UT with the 2.3-m Bok Telescope at the Kitt Peak National Observatory. The comet was at heliocentric distance $\left(r_{h}\right)$ of $1.74 \mathrm{AU}$ and $1.71 \mathrm{AU}$, a geocentric distance $(\Delta)$ of $1.66 \mathrm{AU}$ and $1.69 \mathrm{AU}$, a phase angle of $34.62^{\circ}$ and $34.76^{\circ}$, for each date respectively.

The images were obtained with the 90Prime camera (Williams et al. 2004), a prime focus imager built for the Bok Telescope. At the time of observation, the 90Prime camera utilized a thinned back-illuminated CCD detector with $4064 \times 4064$ pixels with a pixel size of $15.0 \mu \mathrm{m}$. At prime focus the camera pixel scale is $0.45^{\prime \prime}$ which yields a field of view of 30.5 $\times 30.5$ square-arcmin. The instrument was equipped with Cousins/Bessel system broadband $V$ and $R$ filters. Multiple exposures (23 images in $R$ band and 9 images in $V$ band of 30 seconds each) were obtained of the nucleus and coma of the comet with the telescope tracking at the non-sidereal rate corresponding to the predicted motion of the comet provided by JPL Horizond 1 in an airmass range of 1.40 to 1.74 .

All images were corrected for overscan, bias and flat-fielding with standard IRAF2 routines. The data was photometrically calibrated using eight field stars of various spectral types with known $V$ and $R$ magnitudes selected from the Naval Observatory Merged Astrometric Dataset (NOMAD) catalog (Zacharias et al. 2004) on the same CCD amplifier as the comet. The standard deviation of the photometric $V$ and $R$ zero points derived from the average of the field stars is of order $1 \%$ and no color corrections for spectral type were applied. The average nightly seeing was $\sim 2.2^{\prime \prime}$ in both bands. A single 30 sec exposure in

\footnotetext{
${ }^{1}$ http://ssd.jpl.nasa.gov/horizons.cgi

${ }^{2}$ IRAF is distributed by the National Optical Astronomy Observatory, which is operated by the Association of Universities for Research in Astronomy (AURA) under cooperative agreement with the National Science Foundation.
} 
the $R$ band obtained on 2014 June 04.24 UT is shown in Fig. 1.

\subsection{SOFIA}

Mid-infrared (mid-IR) spectrophotometric observations of comet C/2012 K1 (PanSTARRS) were obtained using the Faint Object InfraRed CAmera for the SOFIA Telescope (FORCAST; Herter et al. 2012) mounted at the Nasmyth focus of the 2.5-m telescope of the SOFIA Observatory (Young et al. 2012; Gehrz et al. 2009). The data were acquired over a series of four flights, originating from Palmdale, CA at altitudes of $\simeq 11.89 \mathrm{~km}$ in 2014 June, that were conducted as part of our SOFIA Cycle 2 programs to observe comets (P.I. Woodward, AOR_IDs 01_001 and 02_0002). Details of all SOFIA observations and the orbital parameters of comet C/2012 K1 (Pan-STARRS) at those epochs are summarized in Table 1.

FORCAST is a dual-channel mid-IR imager and grism spectrometer operating from 5 to $40 \mu \mathrm{m}$. Light is fed to two $256 \times 256$ pixel blocked-impurity-band (BIB) arrays, each with a plate scale of $0.768^{\prime \prime}$ per pixel and a distortion corrected field of view of $3.2^{\prime} \times 3.4^{\prime}$. The Short Wavelength Camera (SWC) covers the spectral region from 5 to $25 \mu \mathrm{m}$, while the Long Wavelength Camera (LWC) operates at wavelengths from 25 to $40 \mu \mathrm{m}$. Imaging data can be acquired in either dual channel mode (with some loss of throughput due to the dichroic) or single channel mode.

Imaging observations of C/2012 K1 (Pan-STARRS) in three filters were conducted on the first flight series, prior to three flights dedicated to spectroscopy. Spectroscopic observations of the comet used two grisms, one in the SWC (G111) and one in the LWC (G227), and the instrument was configured using a long-slit $\left(4.7^{\prime \prime} \times 191^{\prime \prime}\right)$ which yields a spectral resolution $R=\lambda / \Delta \lambda \sim 140-300$. The comet was imaged in the SWC using the F197 filter to position the target in the slit. Both imaging and spectroscopic data were obtained using a 2-point chop/nod in the Nod-Match-Chop (C2N) mode with 45" chop and 90" nod amplitudes at angles of $30^{\circ} / 210^{\circ}$ in the equatorial reference frame.

All FORCAST raw image data products were processed using the FORCAST_REDUX Data Pipeline, v1.0.1beta (cf., Clarke. Vacca, \& Shuping 2014), which employed the reduction packages FORCAST_FSPEXTOOL, v1.1.0, and FORCAST_DRIP, v1.1.0. Processing

of the raw spectroscopic data was performed using the same packages, with the exception of FORCAST_DRIP, which utilized v1.0.4. Details of the FORCAST_REDUX Data Pipeline 
can be found in the Guest Investigator Handbook for FORCAST Data Products, Rev. B3

\subsection{SOFIA Imagery and Photometry}

Aperture photometry of the SOFIA image data of comet C/2012 K1 (Pan-STARRS) was performed on the Level 3 pipeline coadded (*.COA) data products using the Aperture Photometry Tool (APT v2.4.7; Laher et al. 2012). At all FORCAST filter wavelengths, the comet exhibited extended emission beyond the PSF of point sources observed with FORCAST under optimal telescope jitter performance 4 The photometry was therefore conducted using a circular aperture centroided on the photocenter of the comet nucleus. We used an aperture of radius 13 pixels, corresponding to 9.984", with a background aperture annulus of inner radius 30 pixels $\left(23.58^{\prime \prime}\right)$ and outer radius of 60 pixels $\left(47.16^{\prime \prime}\right)$. This aperture, which is $\simeq 3 \times$ the nominal point-source FWHM, encompassed the majority of the emission of the comet and coma. Sky-annulus median subtraction (ATP Model B as described in Laher et al. 2012) was used in the computation of the source intensity. The systematic source intensity uncertainty was computed using a depth of coverage value equivalent to the number of coadded image frames. The dominant source of overall uncertainty in the image photometry were image gradients due to imperfect atmospheric background subtraction. The calibration factors (and associated uncertainties) applied to the resultant aperture sums were included in the Level 3 data distribution and were derived from the weighted average of 3 calibrator observations of $\beta$ And (2 each) and $\alpha$ Boo (1 each). The resultant SOFIA photometry is presented in Table 2 ,

Due to turbulence, telescope jitter, and differing chop-nod patterns, i.e., the chopping difference between beams and the nodding of the entire telescope field-of-view (for a for a discussion and illustration of this standard infrared observing technique with SOFIA - see Temi et al. 2014; Young et al. 2012) executed in flight, the multi-filter imagery data could not be used to generate color temperature maps due to the unstable PSF.

During flights primarily devoted to obtaining grism data (\$2.4), images of the comet where obtained through a single filter at 19.7 micron. Figure 2 shows the $19.7 \mu \mathrm{m}$ surface brightness distribution of comet C/2012 K1 (Pan-STARRS) observed on 2014 June 13.17 UT. The nucleus is unresolved and azimuthally symmetric with a radial profile FWHM of $\sim 1.01^{\prime \prime}$

\footnotetext{
${ }^{3}$ https://www.sofia.usra.edu/Science/DataProducts/ FORCAST_GI_Handbook_RevA1.pdf

${ }^{4}$ see http://www.sofia.usra.edu/Science/ ObserversHandbook/FORCAST.html §5.1.2
} 
and the coma is extended and diffuse. Low surface brightness emission extends in a vector direction commensurate with that expected for a dust tail.

\subsection{SOFIA Spectra}

Three temporally distinct spectra of comet C/2012 K1 (Pan-STARRS) were obtained in both grism over a series of flight sequences spanning 6 days (Table 1). Many comets exhibit temporal variability in the infrared over periods of hours (e.g., Wooden et al. 2004) to days at relatively similar heliocentric distances due to coma jets related to nucleus activity and/or nucleus rotation period (e.g., Keller et al. 2007; Gehrz et al. 1995) that produces observable changes in the observed SEDs. Inter-comparison of each SED over this period showed no substantial changes in overall continuum flux densities nor spectral features to within the uncertainty per spectral resolution element. In addition, the $19.7 \mu \mathrm{m}$ aperture photometry suggests also that the level of coma emission did not markedly change (cf., Table 2). Apparently, comet C/2012 K1 (Pan-STARRS) was fairly quiescent in its infrared behavior during this epoch given our signal-to-noise ratio and aperture size $(12,300 \mathrm{~km}$ radius). Thus, the three independent spectra were summed together in pipeline processing to produce an average spectral energy distribution (SED). A 3-point unweighted rectangular smoothing function was applied to this average SED to increase the point-to-point signal-tonoise ratio of the data product used in our thermal model spectral decomposition analysis. The calibrated data products do exhibit a few artifacts near the edges of the 17-27 $\mu \mathrm{m}$ spectral order where a few data points deviate upwards (near $17 \mu \mathrm{m}$ ) or downwards (near $27 \mu \mathrm{m}$ ) from the apparent spectral trend. The spectra of comet C/2012 K1 (Pan-STARRS) are presented in Fig. 3 ,

\section{RESULTS}

Taxonomically comet C/2012 K1 (Pan-STARRS) is a member of the dynamical comet family denoted as nearly isotropic comets (NICs), also commonly referred to as Oort Cloud comets (cf., Dones et al. 2004). The interior composition of the ecliptic comets (ECs) and the NICs likely are preserved during their residence in the Scattered Disk and the Oort Cloud, but their surfaces are subject to various processing effects. Modeling the coma dust properties provides insight into the origin and evolution of dynamic comet families. 


\subsection{Thermal Modeling of the Coma SED}

Thermal modeling of the observed thermal infrared SED of comets obtained using remote sensing techniques enables derivation of coma dust grain properties. In particular, SOFIA (+FORCAST) provides spectroscopic coverage with the G111 grism to the region 9-12 $\mu \mathrm{m}$ which contains features from amorphous and crystalline silicates (e.g., $11.2 \mu \mathrm{m})$ and organic species (e.g., PAHs). The G227 grism spans 17.6-27.7 $\mu \mathrm{m}$, encompassing discrete resonances from crystalline silicates as well as spectral signatures from carbonates and phyllosilicates, putatively argued to be extant in comets (Lisse et al. 2006). The SED slope at long thermal ( $\gtrsim 15 \mu \mathrm{m}$ ) wavelengths provides constraints on the abundance of the larger grain population in the coma. Observations in these spectral regimes are key to ascertaining the origins of silicates within the solar protoplanetary disk, and placing early solar disk evolution within the context of other circumstellar disks observed today through comparison to model and laboratory data (cf., Lindsay et al. 2013; Koike et al. 2010).

Modeling the mid-IR SED of C/2012 K1 (Pan-STARRS) yields estimates of the coma grain properties. We constrain the grain parameters by chi-squared fitting thermal emission models to the observed spectrum. The grain parameters included in the modeling are size distributions (n(a) da), porosity, the crystalline mass fraction (i.e., the fraction of the coma silicate grains that are crystalline), and relative material abundances. The dust temperature is calculated assuming thermal equilibrium of the grains; wherein the composition (mineralogy), size, and heliocentric distance determine the temperature of the grains.

Comet grains are dominated in composition by a handful of silicate-type materials (Hanner \& Zolensky 2010; Wooden 2008): Mg-Fe olivine- and pyroxene-types in amorphous (glassy) forms and their crystalline $\mathrm{Mg}$-end-members forsterite $\left(\mathrm{Mg}_{2} \mathrm{SiO}_{4}\right)$ and enstatite $\left(\mathrm{MgSiO}_{3}\right)$. Cometary aggregates also contain organics (Sandford et al. 2006) or amorphouscarbon-like materials (Matrajt et al. 2008; Formenkova 1999) that may be the glue that holds the amorphous and crystalline materials together (Flynn et al. 2013; Ciesla \& Sandford 2012). Our model (Harker et al. 2002, and references therein) uses five materials: amorphous olivine and amorphous pyroxene with broad 10, 18, and $20 \mu \mathrm{m}$ emission features, amorphous carbon with featureless emission, and crystalline olivine (Mg-rich) and orthopryoxene with narrow peaks. Broad and narrow resonances near 10 and $20 \mu \mathrm{m}$ are modeled by warm chondritic (50\% Fe; $50 \% \mathrm{Mg}$ ) amorphous silicates (i.e., glasses) and strong 11.25, 19.5, and $24 \mu \mathrm{m}$ narrow features from cooler Mg-rich crystalline silicate materials.

The amorphous carbon component in our dust model is representative of several key dust species - e.g., elemental carbon dust (Fomenkova et al. 1994), an organic component with $\mathrm{C}=\mathrm{C}$ bonds, identified by XANES spectra near $285 \mathrm{eV}$ that can include amorphous

carbon (Flynn et al. 2013, 2003; Wirick et al. 2009), and possibly other carbonaceous grains; 
however, overall model results do not depend on this degeneracy. We do not specifically include Fe-Ni sulfides (such as pyrrhotite or troilite) in our models nor carbonates or phyllosilicate-rich materials. The latter materials have not been detected in track analysis of Stardust samples (Nakamura-Messenger et al. 2011; Wooden 2008; Zolenskv et al. 2008) nor are they unequivocally evident in remote sensing data (Bursentova et al. 2012; Woodward et al. 2007). Phyllosilicates, specifically smectites including montmorillonite, chlorite, and serpentine, have $18-23 \mu \mathrm{m}$ resonances that worsen spectral fitting of comet C/1995 O1 (Hale-Bopp) (Wooden et al. 1999). Hybrid IDPs may contain up to $10 \%$ smectite (Nakamura-Messenger et al. 2011). Smectitie is spectrally distinguishable from amorphous anhydrous olivine-type and amorphous pyroxene materials (Nakamura-Messenger et al. 2011; Wooden et al. 1999), yet it is not required for spectral decomposition.

While FeS-type grains are present in IDPs (Bradley \& Dai 2000), meteoritics samples, and comets grains, such as Wild 2 (Heck et al. 2012; Zolensky et al. 2008; Velbel \& Harvev 2007), our SOFIA spectra (Fig. 3) do not exhibit the broad $23 \mu \mathrm{m}$ spectral features often associated with fine-grained FeS (Bursentova et al. 2012; Min et al. 2005; Hony et al. 2002; Keller et al. 2002). Larger FeS particles would be spectrally indistinguishable from larger amorphous carbon particles at mid- to far-IR wavelengths, yet robust optical constants spanning visible through the far-IR are lacking for FeS due to measurement challenges of an inherently extremely absorbing material (L. Keller, private comm.). Thus, thermal modeling of FeS gains is uncertain. Stardust samples appear to be richer in FeS and poorer in carbonaceous matter (Joswiak et al. 2012), so there is no basis as yet to make an assumption about the relative abundance of FeS and amorphous carbon-like materials in comet comae. Hence we presume that the majority of absorbing materials in cometary dust re-radiating the observed infrared SED is dominated by olivine, pyroxene, and carbonaceous (amorphous carbon-like) materials (Brownlee 2014; Wooden 2008; Zolensky et al. 2008). This presumption provides a foundation for comparing compositional similarities and diversities of comet dust composition derived from thermal models.

The best-fit chi-square model results are summarized in Table 3. The model fit to the observed grism spectra with the corresponding spectral decomposition of grain components is presented in Fig. 4. Mineralogically, the grains in the coma of C/2012 K1 (Pan-STARRS) are dominated by amorphous materials, especially carbon. Our models produce a Hanner (modified-power law) differential grain size distribution (HGSD) 5 peaking with grains of radii $a_{\text {peak }}=0.6 \mu \mathrm{m}$, indicating relative moderately larger grains are present, and the grain power-law slope $N=3.4$. In a HGSD the small radii grains at the peak of the grain size

\footnotetext{
${ }^{5}$ This power law (in grain radii, $a$ ) is defined as $n(a) d a \equiv\left(1-a_{o} / a\right)^{M}\left(a_{o} / a\right)^{N}$; where $a_{o}=0.1 \mu \mathrm{m}$ and $a_{\text {peak }}=a_{o}(N+M) / N$.
} 
distribution dominate the surface area and the flux density.

Grains in comets are likely fractal porous aggregates Schulz et al. (2015). The grain porosity ( $P$ versus the dust radius $a)$, parameterized by $D$, is defined as $P=(a / 0.1 \mu \mathrm{m})^{(\mathrm{D}-3)}$ with $D=3$ for solid and $D=2.5$ for highly porous grains (Woodward et al. 2011). Grains in the coma of $\mathrm{C} / 2012 \mathrm{~K} 1$ (Pan-STARRS) also are solid (the fractal porosity parameter $D=3.0)$. Solid grains are not unusual, $65 \%$ of the Stardust tracks are carrot-shaped from solid terminal particles (Hörz et al. 2006). The sub-micron sized silicate-to-carbon ratio derived from our models is $0.80_{-0.20}^{+0.25}$. The uncertainty in the parameters derived from the thermal models are at the $95 \%$ confidence level.

\subsection{The $10 \mu \mathrm{m}$ Silicate Emission Feature}

The $10 \mu \mathrm{m}$ silicate feature in comet C/2012 K1 (Pan-STARRS) is quite weak compared to comets like C/1995 O1 (Hale-Bopp) or 17P/Holmes (e.g., Wooden et al. 1999; Watanabe et al. 2009). Following (Sitko et al. 2004), at $10.5 \mu \mathrm{m}$ we find the silicate emission (defined as $\left[F_{10} / F_{\text {continuum }}^{B B}\right]$ ) is $1.18 \pm 0.03$ above a blackbody curve fit to the observed grism spectra continua longwards of $12.5 \mu \mathrm{m}$. The best-fit blackbody is $T_{b b}=215.32 \pm 0.95 \mathrm{~K}$ (using Gaussian weighted errors) and color excess, defined as $\mathrm{T}_{b b}($ fit $) /\left(278 \mathrm{~K} r_{h}^{-0.5}\right)$ is $=0.992 \pm 0.004$. The normalized $\left(F_{\lambda} / F_{\lambda, T}\right)$ SED in the region near the silicate feature at $10 \mu \mathrm{m}$ is presented in Fig. 5. Typically data near $8 \mu \mathrm{m}$ are used to establish the blue-continua $(\lambda \lambda 7.7-8.4 \mu \mathrm{m})$. Our estimate of the local $10 \mu \mathrm{m}$ continua may yield slightly lower temperatures than an estimate that included $8 \mu \mathrm{m}$ photometry.

The large value of $a_{\text {peak }}$ inferred from the thermal modeling of the observed SED of comet $\mathrm{C} / 2012 \mathrm{~K} 1$ (Pan-STARRS) in 2014 June is commensurate with the weak $10 \mu \mathrm{m}$ silicate feature. Smaller grains $\left(a_{\text {peak }} \lesssim 0.3 \mu \mathrm{m}\right)$ produce higher contrast silicate features. Grains of greater porosity also produce higher contrast silicate features in the $10 \mu \mathrm{m}$ band. Long period NICs have 'typical' HGSD slopes of $3.4 \lesssim N \lesssim 3.7$ and silicate-to-amorphous carbon ratios $\gg 1$. The grain size distribution slope of $\mathrm{C} / 2012 \mathrm{~K} 1$ (Pan-STARRS), $N=3.4$, is not atypical. The preponderance of larger sub-micron grains $\left(a_{\text {peak }}=0.6 \mu \mathrm{m}\right)$ in the coma of comet $\mathrm{C} / 2012 \mathrm{~K} 1$ (Pan-STARRS) results in cooler radiating dust that contributes to the 'continuum' under the $10 \mu \mathrm{m}$ silicate feature and to the far-infrared flux density (see Fig. (4). The sub-micron mass fraction is dominated by amorphous carbon grains. Amorphous carbon has a featureless emission spectrum that extends through the $10 \mu \mathrm{m}$ region, so low

amorphous silicate-to-carbon ratio also can weaken the silicate feature strength (Wooden 2008; Wooden et al. 2004). 


\subsection{The Silicate Crystalline Mass Fraction}

The mass fraction of silicate sub-micron grains that are crystalline in comet comae is a keystone for models of early planet-forming processes (Bockelée-Morvan et al. 2002; Ciesla 2007; Hughes \& Armitage 2010). This fraction is defined as

$$
f_{\text {cryst }}^{\text {silicates }} \equiv \sum_{x=1}^{n} \frac{m_{\text {cryst }, x}}{\left(m_{\text {cryst }, x}+m_{\text {amorphous }, x}^{\text {silicates }}\right)}
$$

where $\mathrm{m}_{x}$ is the mass of species $x$. Crystalline species in comet grains provide a record of the high temperature process that formed dust in the inner disk of the solar system and the large scale mixing that transported these hot nebular products to the cold comet forming zones. Crystals, their composition (e.g., Wooden 2008) and shape (Lindsay et al. 2013) trace inner-solar disk conditions (e.g., Ogliore et al. 2011) and offer a view into the earliest planet-forming processes that occurred in our early Solar System.

Crystals from the inner disk were transported out to the comet-forming regime and mixed with "amorphous" silicates (cf. Cielsa 2011). The "amorphous" silicates are thought to be outer disk materials that probably were inherited from the ISM Brownlee 2014; Watson et al. 2009; Kemper et al. 2004, 2005; Li \& Draine 2001) in the infall phase of the disk. They have non-stoichiometric compositions (GEMS-like, Matsuno et al. 2012; Bradley \& Dai 2004; Bradley et al. 1999, and references therein) that include the compositional ranges of olivine $\left[\left(\mathrm{Mg}_{y}, \mathrm{Fe}_{(1-y)}\right)_{2} \mathrm{SiO}_{4}\right]$, with $\mathrm{y} \approx 0.5$ for amorphous olivine, and $\left[\left(\mathrm{Mg}_{x}, \mathrm{Fe}_{(1-x)}\right) \mathrm{SiO}_{3}\right]$ with $\mathrm{x} \approx 0.5$ for amorphous pyroxene-type materials. Crystals are identified by narrow IR emission features (e.g., 11.2, 19, 23.5, 27.5, $33 \mu \mathrm{m}$ ) superposed on an underlying thermal continuum in remote sensing spectra. Crystalline silicates have been detected using remote sensing techniques in the dust comae of all comet classes including C/1995 O1 (Hale-Bopp) (Wooden et al. 1999; Harker et al. 2002, 2004a) the Deep Impact coma of 9P/Tempel 1 (Harker et al. 2005; Lisse et al. 2006; Harker et al. 2007), the fragmentation outburst of 17P/Holmes (Reach et al. 2010), and several other comets (Kelley \& Wooden 2009; Woodward et al. 2011). Amorphous silicates are also detected in these comets as well. Crystalline silicates are found in abundance in the Stardust samples of 81P/Wild 2; however, the amorphous grains are difficult to identify (Ishii et al. 2008) and may be limited to the smallest dust grains (Brownlee 2014).

Crystalline silicates are rare in the ISM; however, they account for $\lesssim 2.2 \%$ of the total silicate component in the direction of the Galactic Center (Kemper et al. 2004, 2005) and $\lesssim 5 \%$ along other lines-of-sight (Li \& Draine 2001). Solar System crystalline silicates

detected in comets must be formed in the early stages of our disk's evolution (Brownlee 
2014). Crystalline silicates require $\mathrm{T} \gtrsim 1000 \mathrm{~K}$ to form through either gas phase condensation or annealing of amorphous (glassy) silicate grains (Wooden 2008; Wooden et al. 2005; Davoisne et al. 2006; Henning 2003; Fabian et al. 2000) implying that the crystalline silicates must have been processed in the disk near the young Sun or in shocks out to a maximum distance of 3 to 5 AU (Harker \& Desch 2002; Wehrstedt \& Gail 2008). Post-formation, they were transported radially outward into the comet-formation zones (Charnoz \& Morbidelli 2007) - a process that is apparently ubiquitous in observations of external protoplanetary disks Olofssson et al. (2010). Glassy silicate spherules (GEMS) and crystals are seen in aggregates in cometary IDPs. Large 'terminal particle' crystals and sub-micron crystals (crys-

tallites) are components of aggregate grains captured in Stardust samples (Brownlee et al. 2012, 2006; Zolensky et al. 2008, 2006).

Thus to first order, the diversity of comet dust properties reflects the temporal and radial gradients in our Solar System's early history and similarities and differences in dust characteristics, including $f_{\text {cryst }}$, may provide observational tests of of planetary migration models within the early solar system during the epoch of planet formation that resulted in a variety of small body dynamical populations. We find a that the silicate crystalline mass fraction in comet $\mathrm{C} / 2012 \mathrm{~K} 1$ (Pan-STARRS) is $f_{\text {cryst }}=0.20_{-0.10}^{+0.30}$. This range is similar to that found for comet $\mathrm{C} / 20007 \mathrm{~N} 3$ (Lulin), $f_{\text {crust }}=0.48 \pm 0.06$ (derived from the mass fractions presented in Table 3 of Woodward et al. 2011, and Eqn. 1 of \$3.3) which also exhibited a weak $10 \mu \mathrm{m}$ silicate emission feature.

\subsection{EC and NIC Dust Characteristics}

As a result of giant planet migration, some comet nuclei were dynamically scattered into the Oort cloud to be exposed to the Galactic environment, whereas those bodies comprising the bulk of the ECs population have nuclei exposed and processed (at depths ranging from $\mathrm{mm}$ to few $\mathrm{cm}$ ) by solar insolation, space weathering, and heliocentric activity variations (sublimation of $\mathrm{CO}, \mathrm{CO}_{2}$; crystallization of water and other ices) which affects materials lofted into the comae. Although the interior compositions of ECs and NICs likely are preserved, their surfaces have differing processing histories.

Typically, active comets (arising from a population of NICs dynamically derived from the Oort Cloud and moving on long-period orbits) exhibit high contrast $10 \mu \mathrm{m}$ silicate features. In contrast, short-period ECs (i.e., Jupiter-family comets) have, on average, lower $10 \mu \mathrm{m}$ silicate features strengths (Sitko et al. 2004), and are thought to have lower activities (cf., the active area and active fraction measurements of A'Hearn et al. 1995). For decades, the low-activity of ECs has been attributed to the accumulation of a rime of insulating 
larger grains that were launched on non-escape orbits (Jewitt 2007). Thermal models that fit observed infrared spectra of comets reveal that high contrast silicate features arise from comae having a preponderance of sub-micron grains (Harker et al. 2002; Hanner et al. 1994). Comae without these sub-micron grains have weaker silicate features. In individual comets, variations in the silicate feature strength have been seen on short time scales corresponding to the aperture-crossing times of jets or coma features (Wooden et al. 2004; Harker et al. 2005, 2007; Gicquel et al. 2012). These variations are best explained by changes in the differential grain size $(\mathrm{n}(\mathrm{a})$ da) or fluctuations in the silicate-to-carbon grain ratio.

Differences between EC and NIC coma grain populations may arise from the surface layers EC nuclei being "processed" or weathered (e.g., Li et al. 2015). Processing of ECs surfaces may result from their frequent perihelion passages that decreases surface volatiles and small grains and leads to the creation of rimes and dust mantles. Evidence suggesting such processing occurs over millennia may be found in the analysis of material excavated from comet 9P/Tempel 1 by Deep Impact: the dust grains in the ejecta were smaller than those in the ambient coma (Harker et al. 2007) and the immediate comet surface contained a layer of carbon rich grains (Sugita et al. 2005) and a dust mantle comprised of compact $20 \mu \mathrm{m}$ sized dust aggregates (Kobayashi et al. 2013). However, this conjecture is not definitive as it unknown whether or not the impact location reflects the global surface dust properties of the nucleus. In ECs, the coma $10 \mu \mathrm{m}$ silicate feature strengths are low (Kelley \& Wooden 2009) and the dust production rates are modest. However, when EC nuclei have either fragmented (i.e., 73P/SW3, Harker et al. 2011; Sitko et al. 2011), explosively released materials from subsurface cavities (i.e., 17P/Holmes, Reach et al. 2010), or have had subsurface materials excavated from depth (i.e., the 9P/Tempel 1 Deep Impact encounter, Harker et al. 2005) the IR SEDs exhibit $10 \mu \mathrm{m}$ relatively strong silicate feature emission $(\gtrsim 1.2)$ arising from a population of sub-micron size silicate grain species. Whether or not the strong $10 \mu \mathrm{m}$ silicate features arise from the release of sub-micron sized grains or the disruption of loose aggregates of fine particles (e.g., through gas-pressure disruption or impact fragmentation) is not known. Indeed the silicate feature in $9 \mathrm{P} /$ Tempel 1 changed from a EC-like spectra to NIC-like spectra immediately after Deep Impact event, returning to an EC-like state several tens of hours later (cf., Harker et al. 2005).

NICs are canonically considered to be more pristine with higher surface volatile abundance (cf., Wooden 2008) - the effects of dwell time in the Galactic environment being more benign. Also, NICs are often considered a homologous population lacking significant nucleus evolution. Inner solar system apparitions of these comets frequently result in brilliant comae, with large dust production rates and pronounced silicate feature emission at IR wavelengths. It is not entirely clear whether the highly active nuclear regions of NICs can spawn small sub-micron grains responsible for the silicate feature emission, either by heritage or by frag- 
mentation induced within the gas acceleration zone. However, whether comet evolution, such as processing in the Galactic environment, can be ignored when comparing the Oort cloud comet dust composition (including that expressed in $f_{\text {cryst }}$ ) is an open question.

Table 4 present estimates of $f_{\text {cryst }}$ and select characteristics of the dust derived from thermal modeling of the mid-IR SEDs for a set of well-studied Oort cloud and "disrupted" Jupiter-family comets. The crystalline silicate fraction ranges appreciably, from $\sim 10 \%$ to $\sim 80 \%$. The compositional similarity suggests that Oort cloud and Jupiter-family comets have common origin sites within the early solar system (an argument that parallels that derived from volatile composition studies, A'Hearn et al. 2012), but the range of $f_{\text {cryst }}$ values in Oort cloud comets suggest this class may be sampling a particular region that is not represented in the Jupiter-family members. This inference is intriguing; however, limited in robustness as any tentative conclusions are based on a limited sample size. Large sample sizes are required to substantiate or vitiate these trends.

Comet C/2012 K1 (Pan-STARRS) and C/2007 N3 (Lulin) have modest mean values for $f_{\text {cryst }}(\lesssim 48 \%$ ), low silicate-to-carbon ratios, and grain size distributions that peak at large radii, $\gtrsim 0.6 \mu \mathrm{m}$. The $10 \mu \mathrm{m}$ silicate feature is weak and/or absent in these NICs. Perhaps these bodies represent a population of more carbon dominated bodies, similar to the dark organic KBOs, whose surfaces are devoid of small grains. Indeed the low albedo (see 93.6 ) of C/2012 K1 (Pan-STARRS) and the dominance of amorphous carbon grain materials maybe providing clues.

\subsection{Dust Production Rates}

The radial profile of comet C/2012 K1 (Pan-STARRS) was plotted to assess the quality of the data for calculating a dust production rate near the epoch of our SOFIA observations. The radial profile of $\mathrm{C} / 2012 \mathrm{~K} 1$ (Pan-STARRS) in the $V$ band shows a deviation from the $1 / \rho$ profile (Gehrz \& Ney 1992), suggesting contamination from gas such as $C_{2}(\Delta=0)$ band(s) near $5141 \AA$. Strong $C_{2}$ emission is present in spectra (McKay et al. 2014, and also A. McKay, priv. comm.) contemporaneous with our optical imagery. We therefore only calculate the dust production in $R$ band. The $R$ band radial profile of $\mathrm{C} / 2012 \mathrm{~K} 1$ (PanSTARRS) is shown in Fig. 6.

To estimate the rate of dust production in comet C/2012 K1 (Pan-STARRS), we utilize the Af $\rho$ quantity introduced by A'Hearn et al. (1984). This quantity serves as a proxy for

dust production and when the cometary coma is in steady state, the value for $A(\Theta) f \rho$ is an aperture independent parameter, 


$$
A(\Theta) f \rho=\frac{4 r_{h}^{2} \Delta^{2} 10^{-0.4\left(m_{\text {comet }}-m_{\odot}\right)}}{\rho}(\mathrm{cm})
$$

where $A(\Theta)$ is four times the geometric albedo at a phase angle $\Theta, f$ is the filling factor of the coma, $m_{\text {comet }}$ is the measured cometary magnitude, $m_{\odot}$ is the apparent solar magnitude, $\rho$ is the linear radius of the aperture at the comet's position $(\mathrm{cm})$ and $r_{h}$ and $\Delta$ are the heliocentric and geocentric distances measured in AU and cm, respectively. To correct our comet measurements for phase angle effects we applied the Halley-Marcus (HM) (Marcus 2007a, b; ; Schleicher et al. 1998) phase angle correction.6 We adopt an interpolated value of 0.3864 (appropriate for the 2014 June $04.24 \mathrm{UT}$ dataset) to normalize $A(\Theta) f \rho$ to $0^{\circ}$ phase angle. Table 5 reports values of $A f \rho=[(A(\Theta) f \rho / \mathrm{HM}]$ at a selection of distances from the comet photocenter in the $R$-band.

In addition to $A f \rho$, we also compute the $\epsilon f \rho$ parameter of comet $\mathrm{C} / 2012 \mathrm{~K} 1$ (PanSTARRS) based on our FORCAST broadband photometry (Table 2). The $\epsilon f \rho$ parameter (defined by Kelley et al. 2013, Appendix A) can be considered to be the thermal emission corollary to the scattered-light-based Af $\rho$ :

$$
\epsilon f \rho=\frac{\Delta^{2}}{\pi \rho} \frac{F_{\nu}}{B_{\nu}}(\mathrm{cm})
$$

where $\epsilon$ is the effective dust emissivity, $F_{\nu}$ is the flux density (Jy) of the comet within the aperture $\rho, B_{\nu}$ is the Planck function $(\mathrm{Jy} / \mathrm{sr})$ evaluated at the temperature $T=$ $T_{\text {scale }}(278 \mathrm{~K}) \mathrm{r}_{\mathrm{h}}^{-0.5}$, where the scaling factor $T_{\text {scale }}=0.99$ based on the $215 \mathrm{~K}$ measured continuum temperature discussed in $\$ 3.2$. Derived values of $\epsilon f \rho$ for comet $\mathrm{C} / 2012 \mathrm{~K} 1$ (PanSTARRS) are presented in Table 2 .

\subsection{Coma Averaged Dust Albedo}

Dust albedo is a basic parameter characterizing the size distribution and physical properties of comet dust that is, surprisingly, infrequently measured. Following the convention of Gehrz \& Ney (1992) the bolometric albedo, $\left(A_{\text {bolometric }} \equiv\left(\right.\right.$ Energy $_{\text {scattered }} /$ Energy $\left._{\text {incident }}\right)$ is

$$
A_{\text {bolometric }} \simeq \frac{f(\Theta)}{1+f(\Theta)}
$$

${ }^{6}$ see http://asteroid.lowell.edu/comet/dustphase.html 
where for comet dust the incident energy is the sum of the energy scattered by the coma plus the total energy of the coma's thermal emission at an observed phase angle $\Theta$ (Suncomet-observer angle). The term $f(\Theta)$ can be determined from fitting the observed spectral energy distribution of the comet with appropriate Planck blackbody functions in the infrared (thermal dust emission) and reflected solar spectra at optical (scattering) wavelengths

$$
f(\Theta)=\frac{\left[\lambda \mathrm{F}_{\lambda}\right]_{\max , \text { scattering }}}{\left[\lambda \mathrm{F}_{\lambda}\right]_{\max , I R}}
$$

where the $\left[\lambda \mathrm{F}_{\lambda}\right]_{\max }$ is the peak of the SEDs in the respective wavelength ranges. Lab experiments and theoretical calculations of the scattered light from particles indicate that the total brightness, color, polarization, and polarization color depend on the optical constants, particle size distribution, structure, and porosity of the dust as well as the solar phase angle (Lindsay et al. 2013; Hadamcik et al. 2007; Kolokolova et al. 2004). The spectral shape of the IR thermal emission provides a direct link with the mineralogy and grain size. Both of these processes provide information on the size and composition of the dust. The scattered light and thermal emission are also connected to one another through the grain albedo, the ratio of the scattered light to the total incident radiation. Because light is not isotropically scattered by comet dust the measured albedo will depend not only on the composition and structure of the dust grains, but also on the phase angle (Sun-comet-observer angle) of the observations.

The coma SED of comet C/2012 K1 (Pan-STARRS) was measured on 2014 June 04 UT using filter photometry at both mid-IR as well as the optical (scattered sunlight) wavelengths. These data enable computation of the coma averaged bolometric albedo (Gehrz \& Ney 1992). Using the integrated flux densities in a circular aperture of radius $9.989^{\prime \prime},\left[\lambda \mathrm{F}_{\lambda}\right]_{\max , I R}$ was derived from the SOFIA photometry by $\chi$-square fitting a blackbody to the mid-IR data using Gaussian weighted errors, resulting in $\mathrm{T}_{b b}=214.04 \pm 14.94 \mathrm{~K}$ with a peak flux of $2.02_{-0.15}^{+0.12} \times 10^{-16} \mathrm{~W} \mathrm{~cm}^{-2}$. The $[\mathrm{V}]$ band photometry is contaminated by gas emission (93.5) . However, the $C_{2}$ bands fall outside the bandpass of the $[\mathrm{R}]$ filter and a $5800 \mathrm{~K}$ blackbody (the Sun) emission peaks near the $[\mathrm{R}]$ filter central wavelength $\left(\lambda_{c}=0.64 \mu \mathrm{m}\right)$ in $\lambda \mathrm{F}_{\lambda}$ $\left(\mathrm{W} \mathrm{cm}{ }^{-2}\right)$ space. Hence, $\left[\lambda \mathrm{F}_{\lambda}\right]_{\text {max scattering }}=3.33 \pm 0.03 \times 10^{-17} \mathrm{~W} \mathrm{~cm}^{-2}$ derived the $[\mathrm{R}]$ band photometry measured in a circular aperture of radius $9.989^{\prime \prime}$ (Table 5). The dust bolometric albedo of comet $\mathrm{C} / 2012 \mathrm{~K} 1$ (Pan-STARRS) is $0.14 \pm 0.01$ at phase angle of $34.76^{\circ}$ (from Eqns. 4 and 5 ).

Kolokolova et al. (2004) reviewed published visual albedos of comets and found only eight 8 comets have measured albedos (excluding comets Kohoutek and Crommelin discussed in Gehrz \& Ney 1992), and all were from the NIC dynamical class. Kelley \& Wooden 
(2009) found only one EC with visual albedo, 21P/Giacobini-Zinner (Pittichová et al. 2008). Recently, the albedos of 73P/Schwassmann-Wachmann 3, 103P/Hartley 2, and C/2009 P1 (Garradd) also have been measured (Meech et al. 2011; Sitko et al. 2011, 2013). Fig. 7 is a compilation of the the bolometric albedo data that exists on comets, including our determination for comet C/2012 K1 (Pan-STARRS). There is considerable scatter for multi-epoch observations of individual comets. Such scatter arises from variations in activity of a comet at different epochs of observation. For example, comet C/1995 O1 (Hale-Bopp), whose data are most scattered, had numerous and fast changing morphological structures (jets, shells, envelopes, e.g., Harker et al. 1997; Woodward et al. 1998). All of these features were characterized by differing size and particle composition (e.g., Rodriguez et al. 1997; Schleicher et al. 1997). Thus, the difference in the dust albedo for the same comet indicates variations in comet activity, specifically development of jets and other morphological features.

The ensemble albedos compiled by Kolokolova et al. (2004) also shows a broad distribution of values for each phase angle. The causes for these latter albedo ranges and the scatter in multi-epoch observations of comets are unclear, but must reside in the physical properties of the comet particles, including the grain size distribution, porosity, grain structure (i.e., prolate spheroids, crystalline needles, etc.), and composition (e.g., Lindsay et al. 2013). However, observations have not yet demonstrated to what extent grain structure or grain compositions are important. To assess these latter aspects, thermal emission models and albedo observations of a additional comets are needed.

\section{SUMMARY}

We discuss the pre-perihelion mid-infrared spectrophotometry and narrow band filter imagery obtained in 2014 June with FORCAST on the NASA SOFIA airborne platform of the dynamically new comet $\mathrm{C} / 2012 \mathrm{~K} 1$ (Pan-STARRS) at a heliocentric distance of $\simeq$ 1.70 AU. The spectral energy distribution of the comet at this epoch exhibits a $10 \mu \mathrm{m}$ silicate feature, $\left[F_{10} / F_{\text {continuum }}^{B B}\right]=1.18 \pm 0.03$ above a blackbody curve $\left(T_{b b}=215.32 \pm 0.95 \mathrm{~K}\right) \mathrm{fit}$ to the spectra continua longwards of $12.5 \mu \mathrm{m}$ which is quite weak compared to comets such as C/1999 O1 (Hale-Bopp) or 17P/Holmes. The coma dust bolometric albedo, $0.14 \pm 0.01$, derived using contemporaneous optical imagery is similar to other comets at the observed phase angle $\left(\sim 35^{\circ}\right)$, while the dust production rate $($ Af $\rho)$ from scattered light observations is $\simeq 5340 \mathrm{~cm}$.

From the observed infrared spectral energy distribution, thermal modeling analysis is used to determine the physical characteristics of the coma dust population and deduce the silicate crystalline mass fraction $\left(0.20_{-0.10}^{+0.30}\right)$ and silicate-to-carbon dust ratio $\left(0.80_{-0.20}^{+0.25}\right)$. We 
find that grains in the coma of $\mathrm{C} / 2012 \mathrm{~K} 1$ (Pan-STARRS) are dominated by amorphous materials, especially carbon, and the differential grain size distribution peaks at radii of $0.6 \mu \mathrm{m}$, the slope of the distribution $N=3.4$, and the grains are solid, having a fractal porosity parameter $D=3.0$. The bulk grain properties of comet $\mathrm{C} / 2012 \mathrm{~K} 1$ (Pan-STARRS) are comparable to other Nearly Isotropic comets (NICs) with weak $10 \mu \mathrm{m}$ silicate features and similar in respect to coma grains seen in the small-set of Ecliptic family comets (ECs) that have fragmented, explosively released subsurface materials, or have had materials excavated from depth.

SOFIA observations of comet C/2012 K1 (Pan-STARRS) and other future comets enables characterization grain properties in the NIC and EC dynamical families. These properties, including dust size, porosity, and composition, relate to grain formation, radial mixing, and particle agglomeration in the proto-solar disk and provide insight to the evolution of the early solar system. As the number of well-studied comets increases at infrared wavelengths (from whence dust properties can be characterized), the fundamental differences between comets originating from different regions and times in the solar system may be eventually discerned.

\section{Acknowledgments}

CEW and his team acknowledge support from Universities Space Research Association (USRA)/NASA contract NAS2-97001. CEW, MSK, DEH also acknowledge support from NASA Planetary Astronomy Program grant 12-PAST12-0016, while CEW and ELR also note support from NASA Planetary Astronomy Program grant NNX13AJ11G. The authors would also like to acknowledge the support and insight of Drs. J. DeBuzier and L. A. Helton of the SOFIA Science Ctr. for their assistance with flight planning and data reduction activities. This work is supported at The Aerospace Corporation by the Independent Research and Development program. We also thank the comments and suggestions of an anonymous referee that improved the clarity of our work.

Facilities: SOFIA (FORCAST), Bok (90Prime) 


\section{REFERENCES}

A'Hearn, M. F., Feaga, L. M., Keller, H. U., et al. 2012, ApJ, 758, 29

A’Hearn, M. F., Millis, R. L., Schleicher, D. G., Osip, D. J., \& Birch, P.V. 1995, Icarus, 118, 223

A'Hearn, M. F., Schleicher, D. G., Feldman, P. D., Millis, R. L., \& Thompson, D. T. 1984, AJ, 89, 579

Berger, E. L., Zega, T. J., Keller, L. P., \& Lauretta, D. S. 2011, Geochim. Cosmochim. Acta, 75,3501

Bockelée-Morvan, D., Gautier, D., Hersant, F., Huré, J.-M., \& Robert, F. 2002, A\&A, 384, 1107

Bradley, J. P., \& Dai, Z. R. 2004, ApJ, 617, 650

Bradley, J. P., \& Dai, Z. 2000, Meteoritics and Planetary Science Supplement, 35, 32

Bradley, J. P., Keller, L. P., Gezo, J., et al. 1999, Lunar and Planetary Science Conference, 30,1835

Brownlee, D. 2014, Ann. Rev. Earth Planet. Sci. 42, 179

Brownlee, D., Joswiak, D., Matrajt, G. 2012, Meteoritics and Planetary Science, 47, 453

Brownlee, D., Tsou, P., Aléon, et al. 2006, Science, 314, 1711

Brusentsova, T., Peale, R. E., Maukonen, D., et al. 2012, MNRAS, 420, 2569

Ciesla, F. J., Sandford, S. A. 2012 Science, 336, 452

Ciesla, F. J. 2011, ApJ, 740, 9

Ciesla, F. J. 2007, Science, 318, 613

Charnoz, S., \& Morbidelli, A. 2007, Icarus, 188, 468

Clarke, M., Vacca, W. D., \& Shuping, R. Y. 2014 i nADASS Conf, Ser., ADASS XXIV, eds. A. R. Taylor \& J. M. Stil [San Francisco, CA: ASP]

Davoisne, G., Djouadi, Z., Leroux, H., et al. 2006, A\&A, 448, L1 
Dones, L., Weissman, P. R., Levison, H. F. \& Duncan, M. J. 2004, in Comets II, eds. M C . Festou, H. U. Keller, and H. A. Weaver, [University of Arizona press: Tucson AZ], p.153ff

Fabian, D., Jager, C., Henning, Th., et al. 2000, A\&A,364, 282

Formenkova, M. N. 1999, Space Sci. Rev. 90, 109

Fomenkova, M. N., Chang, S., \& Mukhin, L. M. 1994, Geochim. Cosmochim. Acta, 58, 4503

Flynn, G. J., Wirick, S., Keller, L. P., et al. 2013, Earth, Planets, and Space, 65, 1159

Flynn, G. J., Keller, L. P., Feser, M., Wirick, S., \& Jacobsen, C. 2003, Geochim. Cosmochim. Acta, 67, 4791

Gehrz, R. D., Becklin, E. E., de Pater, I., Lester, D. F., Roellig, T. L., \& Woodward, C. E. 2009, AdSpR 44, 413

Gehrz, R. D., Johnson, C. H., Magnuson, S. D., \& Ney, E. P. 1995, Icarus, 113, 129

Gehrz, R. D., \& Ney, E. P. 1992, Icarus, 100, 162

Gicquel, A., Bockelée-Morvan, D., Zakharov, V. V., Kelley, M. S., Woodward, C. E., \& Wooden, D. H. 2012, A\&A, 542, 119

Gomes, R., et al. 2005, Nature 435, 466

Hadamcik, E., et al. 2007, Icarus, 190, 459

Hanner, M. S., Lynch, D. K., \& Russell, R. W. 1994, ApJ, 425, 274

Hanner, M. H., \& Zolensky, M. E. 2010, Lecture Notes in Physics, Berlin Springer Verlag, 815,203

Harker, D. E., \& Desch, S. J. 2002, ApJ, 565, L109

Harker, D. E., Woodward, C. E., McMurtry, C. W., et al. 1997, Earth Moon and Planets, 78,259

Harker, D. E., Wooden, D. H., Woodward, C. E., \& Lisse, C. M. 2002, ApJ, 580, 579

-. 2004a, Erratum: ApJ, 615, 1081

Harker, D. E., Woodward, C. E., Wooden, D. H., \& Kelley, M. S. 2004b, AAS, 205, 5612H 
Harker, D. E., Woodward, C. E., \& Wooden, D. H. 2005, Science, 310, 278

Harker, D. E., Woodward, C. E., Wooden, D. H., et al. 2007, Icarus, 190, 432

Harker, D. E., Woodward, C. E., Kelley, M. S., Sitko, M. L., Wooden, D. H., Lynch, D. K., \& Russell, R. W. 2011, AJ, 141, 26

Heck, P. R., Hoppe, P., \& Huth, J. 2012, Meteoritics and Planetary Science, 47, 649

Henning, T. 2003, in Lecture Notes in Physics, Vol. 609, Astromineralogy, eds. T. K. Henning, (Springer-Verlag: Berlin), pp.266

Henning, Th. 2010, ARA\&A, 48, 21

Herter, T. L., Adams, J. D., \& de Buizer, J. M. 2012, ApJ, 749, L18

Hill, P. M. et al. 2001, Pub. Nat. Acad. Sci. 91, No.5, 2182

Hörz, F., Bastien, R., Borg, J., et al. 2006, Science, 314, 1716

Hony, S., Bouwman, J., Keller, L. P., \& Waters, L. B. F. M. 2002, A\&A, 393, L103

Hughes, A. L. H., \& Armitage, P. J. 2010, ApJ, 719, 1633

Ishii, H. A., et al. 2008, Science, 319, 447

Jewitt, D. 2007, in Trans-Neptunian Objects and Comets, Saas-Fee Advanced Course 35, v35, p1 [Springer-Verlag: Berlin]

Joswiak, D. J., Brownlee, D. E., Matrajt, G., et al. 2012, Lunar and Planetary Science Conference, 43, 2395

Laher, R. R., et al. 2012, PASP, 124, 737

Li, J.-Y., Thomas, P. C., Veverka, J., et al. 2015, Highlights of Astronomy, 16, 180

Lindsay, S. S., Wooden, D. H., Harker, D. E., et al. 2013, ApJ, 766, 54

Lisse, C. M., et al. 2006, Science, 313, 635

Kelley, M. S., \& Wooden, D. H. 2009, Planet. Space Sci., 57, 1133

Kelley, M. S., Woodward, C. E., Harker, D. E., Wooden, D. H, Sitko, M. L., Russel, R. W., \& Kim, D. L. 2015a, AAS, 2254, 305K

Kelley, M. S., Fernández, Y. R., Licandro, J., et al. 2013 Icarus, 225, 475 
Kelley, M. S., et al. 2006, ApJ, 651, 1256

Keller, H. U., Küppers, M., Fornasier, S., et al. 2007, Icarus, 191, 241

Keller, L. P., Hony, S., Bradley, J. P., et al. 2002, Nature, 417, 148

Kemper, F., Vriend, W. J., \& Tielens, A. G. G. M. 2004, ApJ, 609, 826

-. 2005, Erratum:, ApJ, 633, 534

Kobayashi, H., Kimura, H., \& Yamamoto, S. 2013, aap, 550, 72

Koike, C., et al. 2010, ApJ, 709, 983

Kolokolova, L., et al. 2004, in Comets II, eds. M. C. Festou, H. U. Keller, \& H. A. Weaver, [U. of Arizona Press, Tucson], p.577

Levison, H. F. 1996, Comet Taxonomy, in ASPC, Vol. 107, ed. T. Rettig \& J. M. Hahn, (ASP: Tucson), pp.173-191

Levison, H. F., et al. 2006, Icarus, 184, 619

Levison, H. F., et al. 2009, Nature, 260, 364

Li, A., \& Draine, B. T. 2001, ApJ, 550, L213

Li, A., \& Greenberg, J. M. 1998, A\&A, 338, 364

Marcus, X. 2007a, International Comet Qrtly April, 39

Marcus, X. 2007b, International Comet Qrtly October, 119

Matrajt, G., Ito, M., Wirick, S., et al. 2008, Meteoritics and Planetary Science, 43, 315

Matsuno, J., Tsuchiyama, A., Koike, C., et al. 2012, ApJ, 753, 141

McKay, A., Kelley, M., Cochran, A., Dello Russo, N., DiSanti, M., Lisee, C., \& Chanover, N. 2014, DPS, 46, 11002

Meech, K., et al. 2011, ApJ, 734, L1

Min, M., Hovenier, J. W., de Koter, A., Waters, L. B. F. M., \& Dominik, C. 2005, Icarus, 179, 158

Nakamura-Messenger, K., Clemett, S. J., Messenger, S., \& Keller, L. P. 2011, Meteoritics and Planetary Science, 46, 843 
Ogliore, R. C., et al. 2011, ApJ, 745, L19

Olofsson, J., et al. 2010, A\&A,, 520, A39

Oort, J. H. 1950, Bull. Astron. Inst. Netherlands, 11, 91

Ootsubo, T., Watanabe, J.-I., Kawakita, H., Honda, M., \& Furusho, R. 2007, Planet. Space Sci., 55, 1044

Pitticová J., Woodward, C. E., Kelley, M. S., \& Reach, W. T. 2008, AJ, 136, 112،

Reach, W. T., Vaubaillon, J., Lisse, C. M., Holloway, M., \& Rho, J. 2010,Icarus, 208, 276

Rodriguez, E., Ortiz, J. L., Lopez-Gonzalez, M. J., et al. 1997, A\&A, 324, L61

Sandford, S. A., Aléon, J., Alexander, C. M. O., et al. 2006, Science, 314, 1720

Schleicher, D. G., Lederer, S. M., Millis, R. L., \& Farnham, T. L. 1997, Science, 275, 1913

Schleicher, D. G., et al. 1998, Icarus, 132, 397

Schulz, R., Hilchenbach, M., Langevin, Y., et al. 2015, Nature, 518, 216

Sitko, M. L., Russell, R. W., Woodward, C. E., et al. 2013, Lunar and Planetary Science Conference, 44, 1154

Sitko, M. L., Lisse, C. M., Kelley, M. S., et al. 2011, AJ, 142, 80

Sitko, M. L., Lynch, D. L., Russell, R. W., \& Hanner, M. S. 2004, ApJ, 612, 576

Stodolna, J., Jacob, D., \& Leroux, H. 2012, Geochim. Cosmochim. Acta, 87, 35

Sugita, S., Ootsubi, T., Kadono, T., et al. 2005, Science, 310, 274

Temi, P., Marcum, P. M., Young, E., et al. 2014, ApJS, 212, 24

Velbel, M. A., \& Harvey, R. P. 2007, Lunar and Planetary Science Conference, 38, 1700

Walsh, K. J., et al. 2011, Nature, 475, 206

Watanabe, J.-I., Honda, M., Ishiguro, M., et al. 2009, PASJ, 61, 679

Watson, D. M., Leisenring, J. M., Furlan, E., et al. 2009, ApJS, 180, 84

Wehrstedt, M., \& Gail, H.-P. 2008, arXiv:0804.3377

Williams, G. G., Olszewski, E., Lesser, M. P., \& Burge, J. H. 2004, Proc. SPIE, 5492, 787 
Williams, G. V. 2015, Observations and Orbits of Comets, Minor Planet Elec. Circ., 2015A10

Wirick, S., Flynn, G. J., Keller, L. P., et al. 2009, Meteoritics and Planetary Science, 44, 1611

Wooden, D. H. 2008, Space Sci. Rev., 138, 75

Wooden, D. H., Harker, D. E., Woodward, C. E., et al. 1999, ApJ, 517, 1034

Wooden, D. H., Woodward, C. E., \& Harker, D. E. 2004, ApJ, 612, L77

Wooden, D. H., Harker, D. E., \& Brearley, A. J. 2005, ASP Conf. Series, v341, eds. A. N. Krot, E. R. D. Scott, \& B. Reipurth. (San Francisco: ASAP), p.774

Wooden, D. H., Woodward, C. E., Kelley, M. S., Harker, D. E., et al. 2011, EPSC Abs. Vol. 6, EPSC-DPS2011-1557

Woodward, C. E., Gehrz, R. D., Mason, C. G., Jones, T. J., \& Williams, D. M. 1998, Earth Moon and Planets, 81, 217

Woodward, C. E., Kelley, M. S., Bockelée-Morvan, D., \& Gehrz, R. D. 2007, ApJ, 671, 1065

Woodward, C. E., et al. 2011, AJ, 141, 181

Woodward, C. E., Russell, R. W., Harker, D. E., Kim, D. L., Cabreira, B., Sitko, M. L., Wooden, D. H., \& Kelley, M. S. 2013, IAUC 9256

Young, E. T., Becklin, E. E., Marcum, P. M., Roellig, T. L., et al. 2012, ApJ, 749, L17

Zacharias, N., Monet, D. G., Levine, S. E., Urban, S. E., Gaume, R., Wycoff, G. L. 2004, Bulletin of the American Astron. Soc. 36, 1418

Zolensky, M. E., Nakamura-Messenger, K., Rietmeijer, F., et al. 2008, Meteoritics and Planetary Science, 43, 261

Zolensky, M. E., Zega, T. J., Yano, H., et al. 2006, Science, 314, 1735 


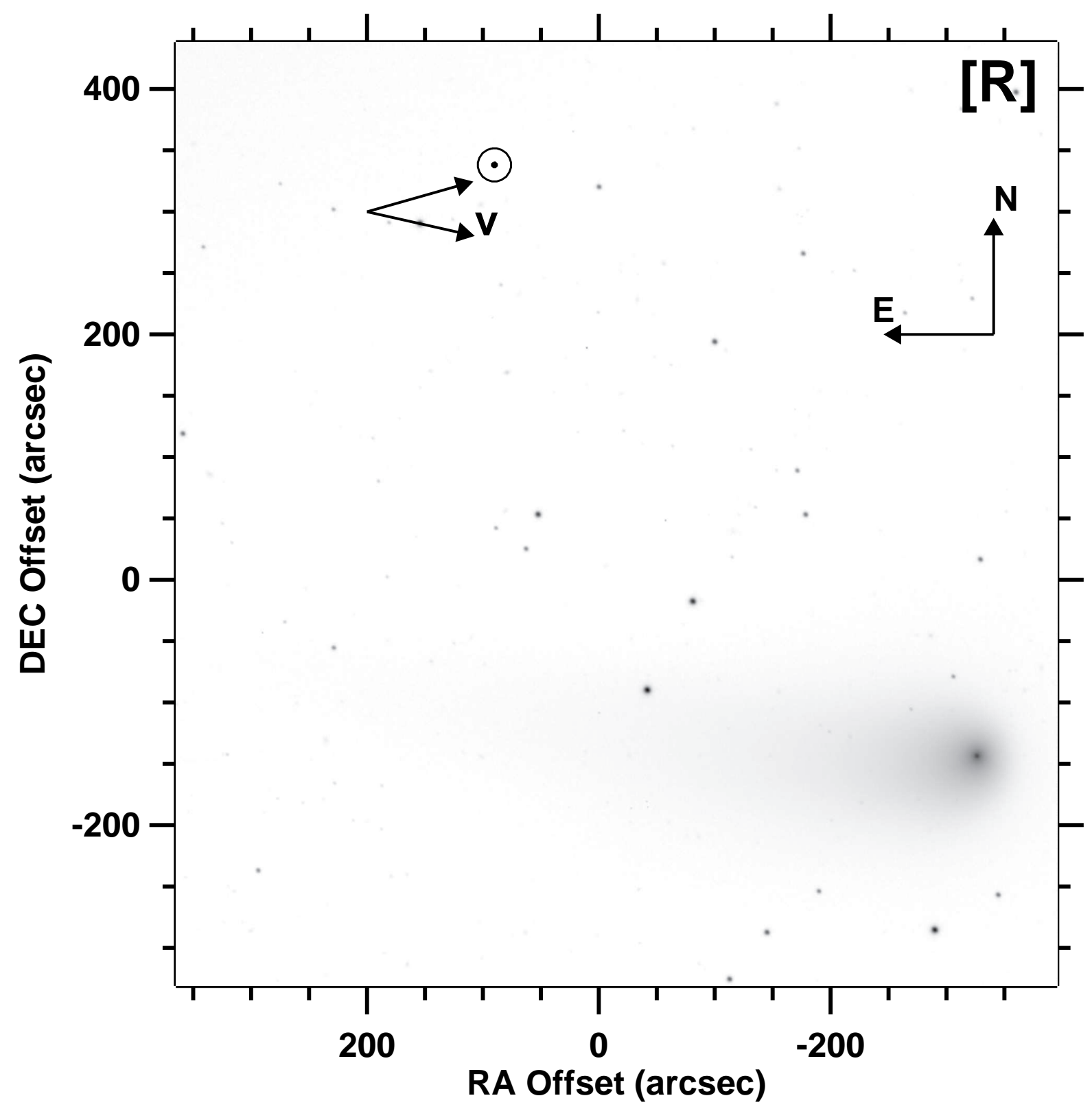

Fig. 1.- The Bok R-band optical images of comet C/2012 K1 (Pan-STARRS) obtained on 2014 June 04.24 UT, with logarithmic greyscale color map. The vector indicating the direction of the comet's motion and the vector indicating the direction toward the Sun are also provided. 


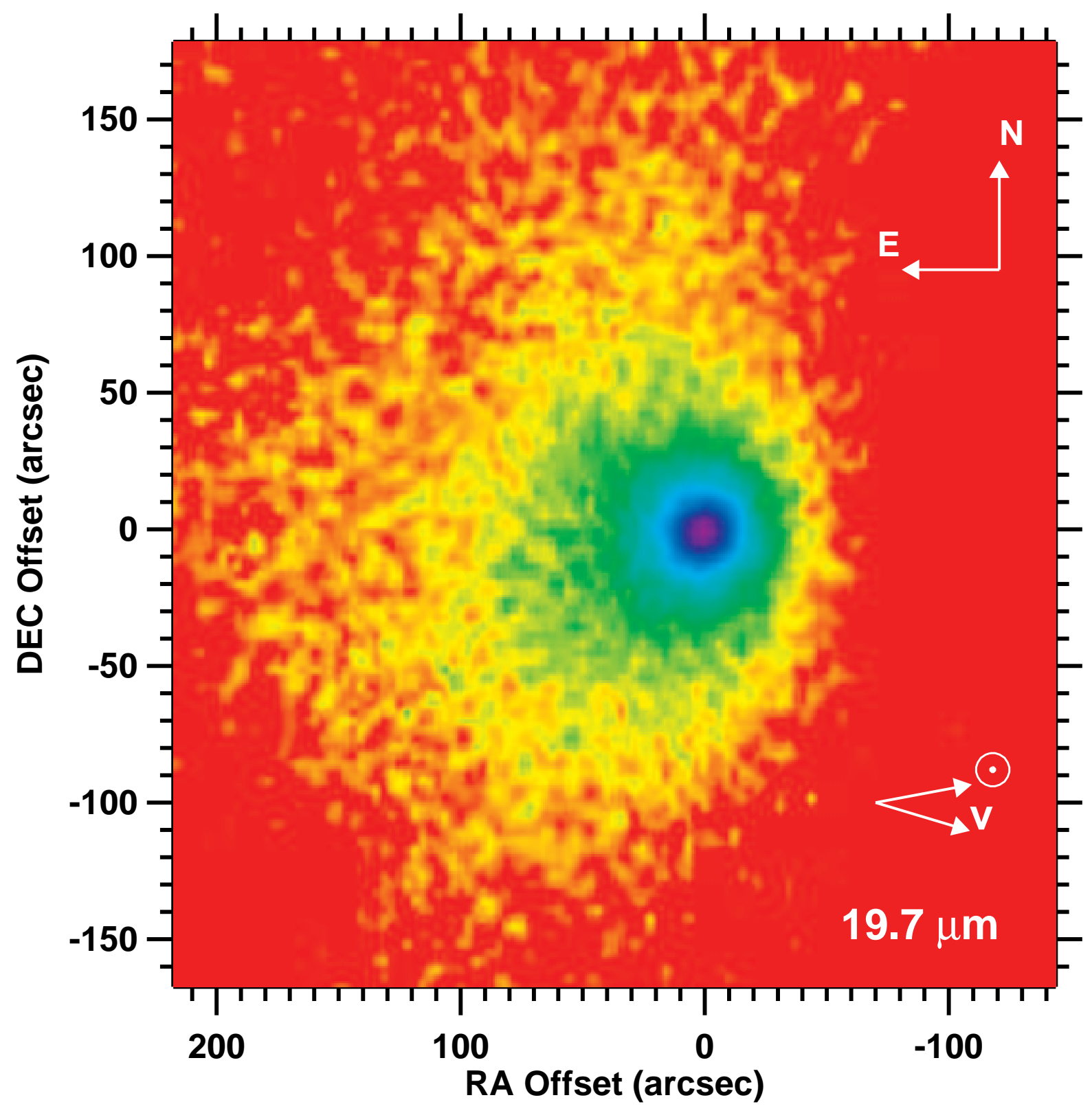

Fig. 2.- The SOFIA $19.7 \mu \mathrm{m}$ image of comet C/2012 K1 (Pan-STARRS) obtained on 2014 June 13.17 UT, with logarithmic color map. The vector indicating the direction of the comet's motion and the vector indicating the direction toward the Sun are also provided. 


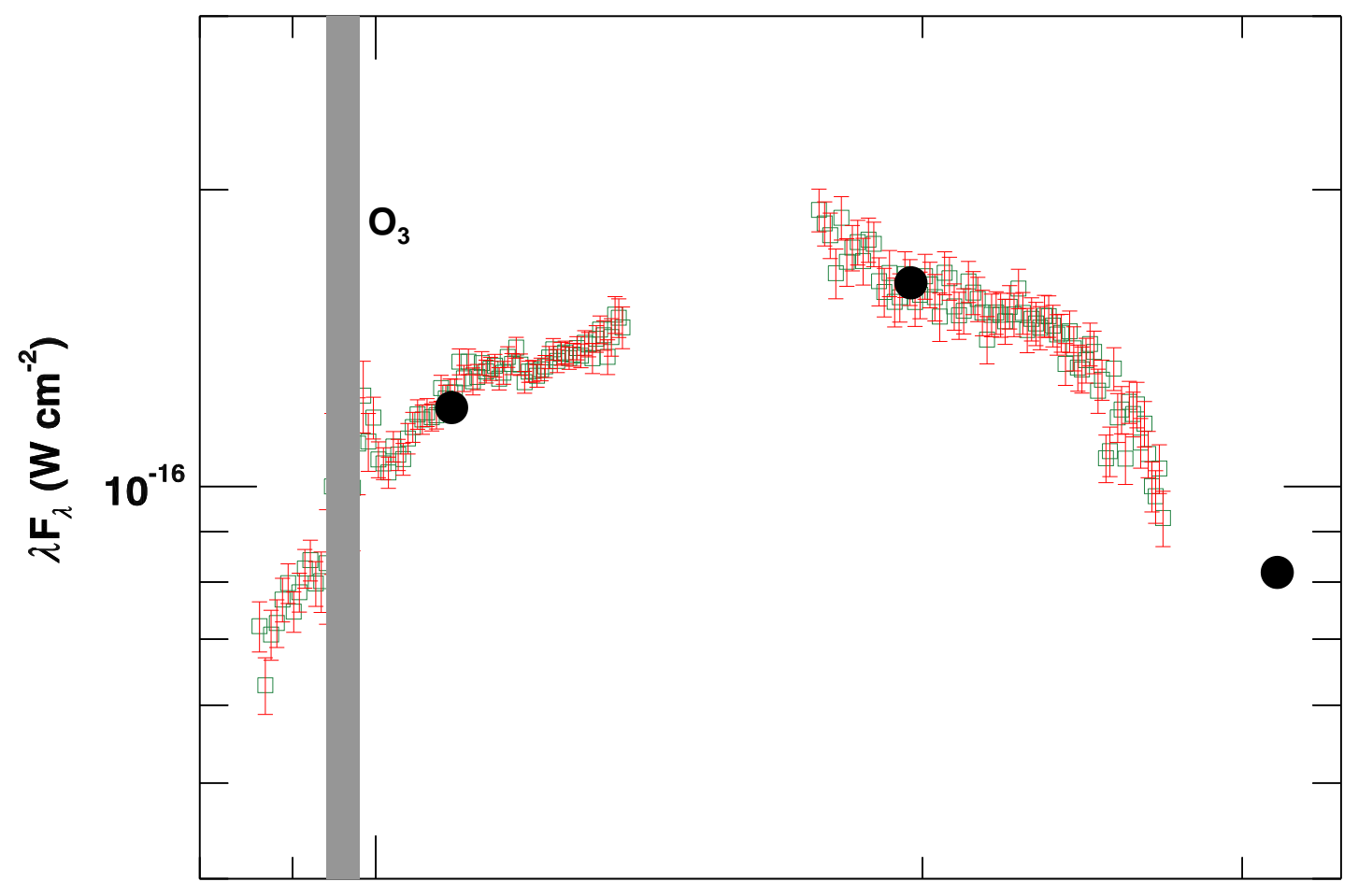

10

\section{Wavelength (micron)}

Fig. 3.- The SOFIA grism observations of comet C/2012 K1 (Pan-STARRS). Grism observations from three flight series where combined to create a single average SED, and a three-point unweighted rectangular smoothing function was applied to the composite spectra to improve the signal-to-noise. The filled circles are the broadband photometric observations obtained on 2015 June 04 UT when each of the filters were observed on a single flight. The photometry is scaled by a factor of 0.75 (which is of the order of absolute photometric calibration uncertainty of FORCAST) to match the flux density of the grism spectra and demonstrate that the shape of the photometric SEDs mimics that of the spectroscopic spectral segments. The vertical grey bar indicates the region of terrestrial ozone absorption. 


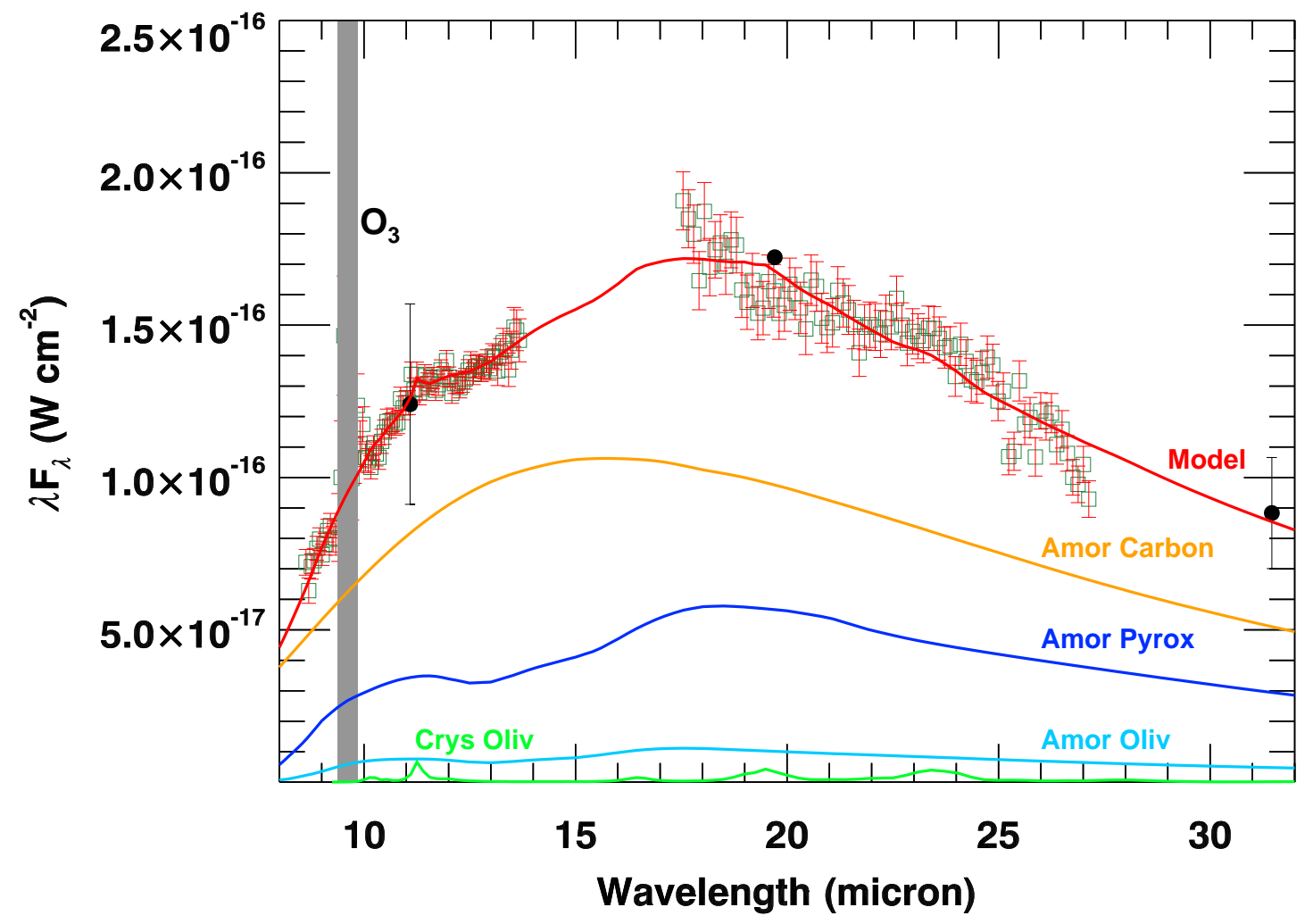

Fig. 4. - The spectral decomposition of the SOFIA grism observations (see Fig. 3) of comet C/2012 K1 (Pan-STARRS) derived from thermal modeling. The filled circles are the broadband photometric observations obtained on 2015 June 04 UT when each of the filters were observed on a single flight. The filter photometry is scaled by a factor of 0.75 (which is of the order of absolute photometric calibration uncertainty of FORCAST) to match the flux density of the grism spectra and demonstrate that the shape of the photometric SEDs mimics that of the spectroscopic spectral segments. The vertical grey bar indicates the region of terrestrial ozone absorption. 


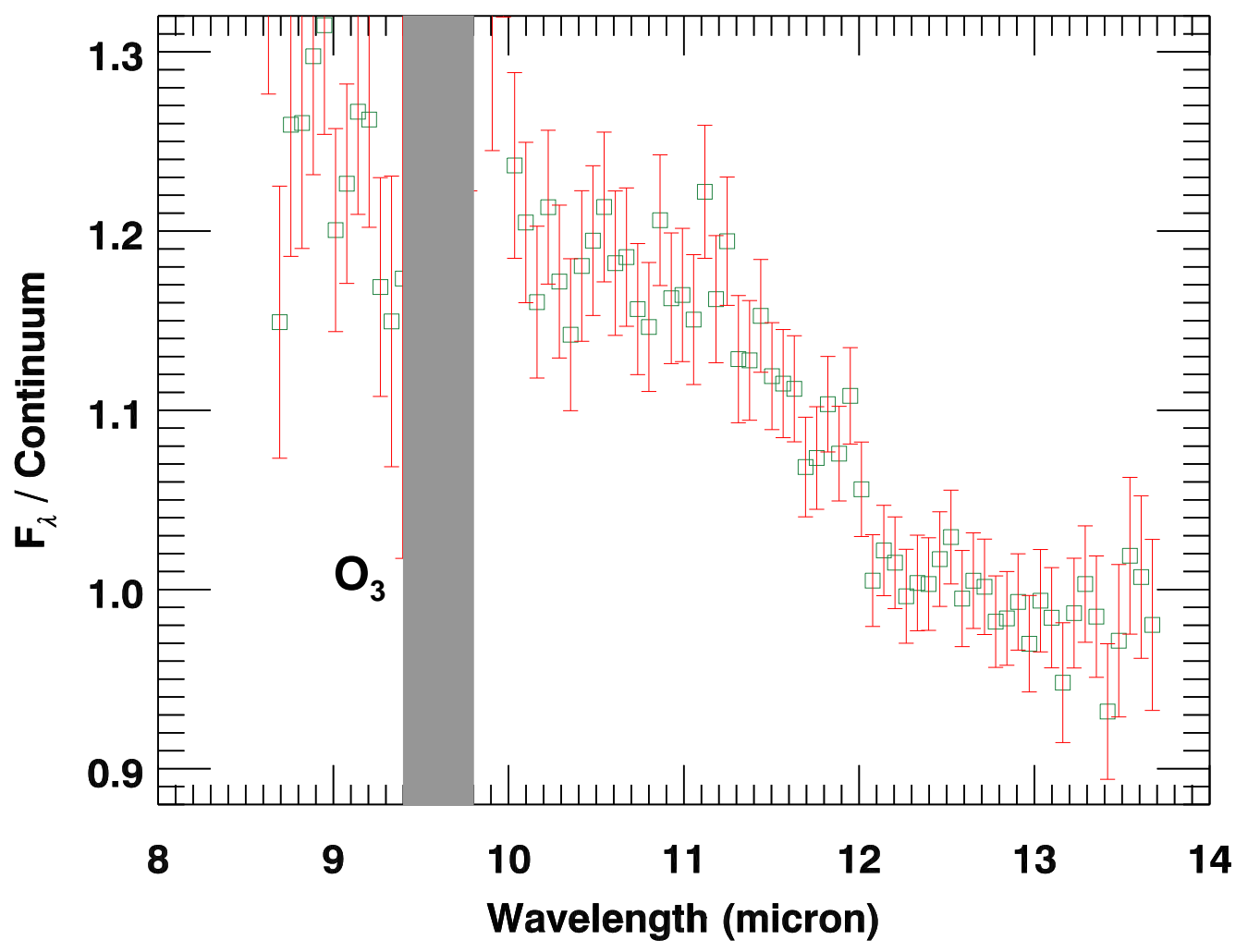

Fig. 5.- The observed SOFIA grism flux density of comet C/2012 K1 (Pan-STARRS) near the $10 \mu \mathrm{m}$ silicate emission feature divided by a $\simeq 215 \mathrm{~K}$ blackbody continuum $\left(F_{\lambda} / F_{\lambda, T}\right)$ to highlight the details of the $10 \mu \mathrm{m}$ silicate feature. The vertical grey bar indicates the region of terrestrial ozone absorption. 


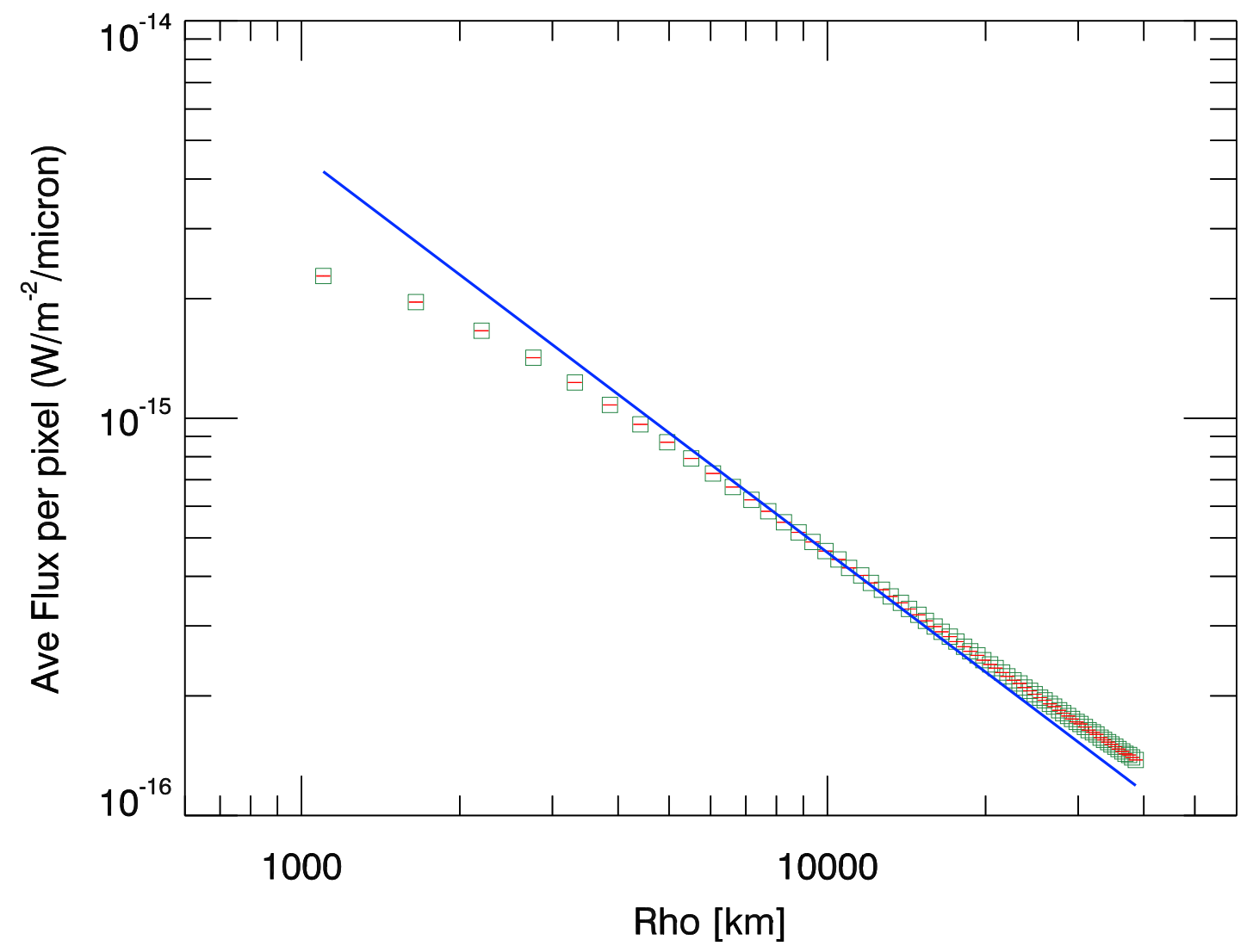

Fig. 6.- Azimuthally averaged radial profile fluxes as a function of linear radius as measured in the $R$ band from the optical photocenter of comet C/2012 K1 (Pan-STARRS) obtained on 2014 June 04.24 UT. The solid blue line denotes a $1 / \rho$ profile. 


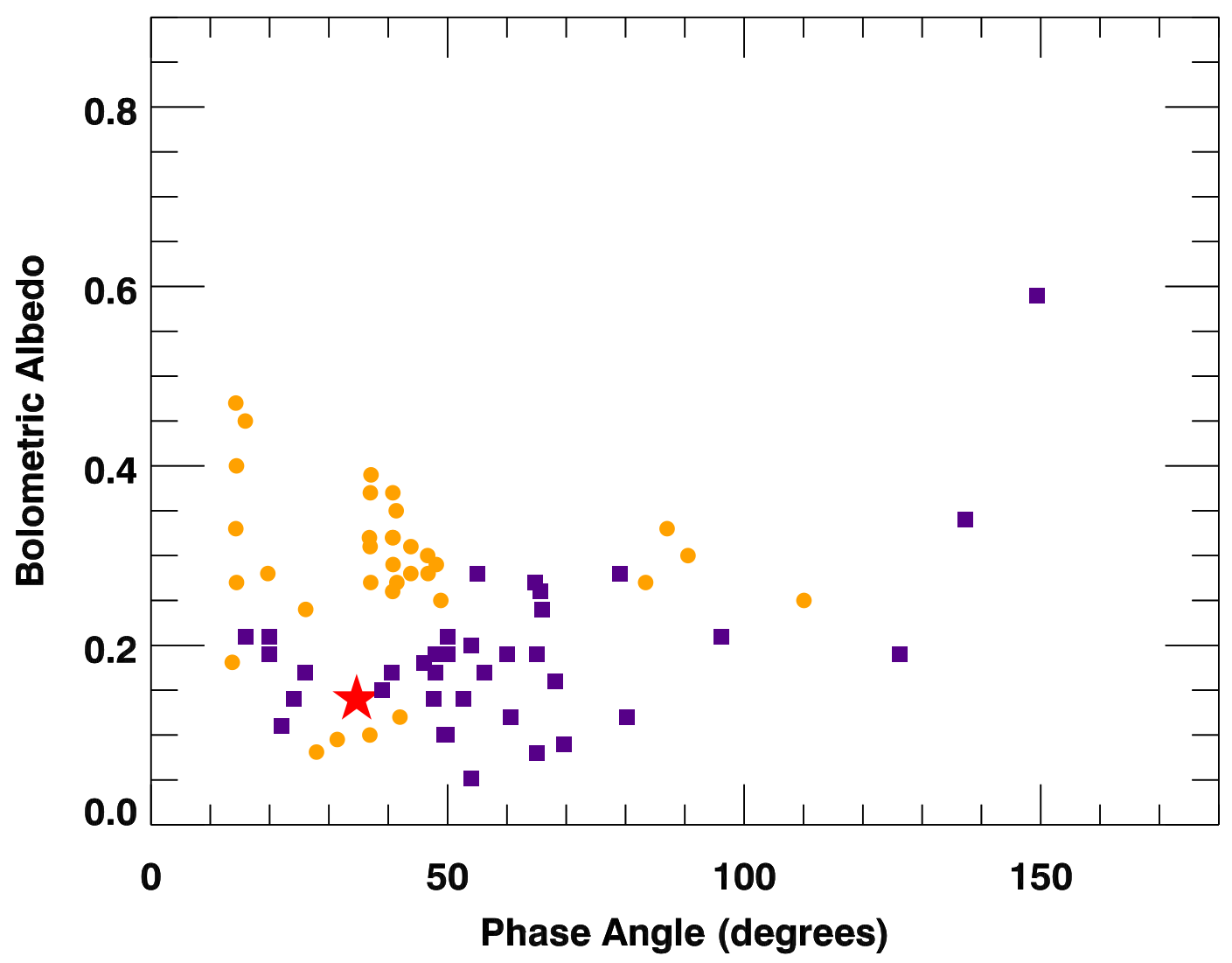

Fig. 7.- The bolometric albedo as a function of phase angle for a sample of Nearly Isotropic Comets (NICs; filled orange circles) and Ecliptic Comets (ECs; filled purple squares) derived from the literature following the prescription of (Gehrz \& Ney 1992), Our measurement of the NIC C/2012 K1 (Pan-STARRS), $0.14 \pm 0.01$ at a phase angle of $34.76^{\circ}$, is indicated by the red star. The phase angles for each comet are obtained from the JPL Horizons ephemerides. 
Table 1. SOFIA Observational Summary - Comet C/2012 K1 (PAN-STARRS)*

\begin{tabular}{|c|c|c|c|c|c|c|c|c|c|}
\hline $\begin{array}{c}\text { Observation } \\
\text { Date } \\
2014 \text { UT } \\
\text { (dd-mm hr:min:s) }\end{array}$ & InstCfg & $\begin{array}{c}\text { Grism } \\
\text { or } \\
\text { Fltr } \\
\lambda_{c} \\
(\mu \mathrm{m})\end{array}$ & $\begin{array}{l}\text { Exp } \\
\text { Time } \\
(\mathrm{sec})\end{array}$ & $\begin{array}{c}\text { Total } \\
\text { On Src } \\
\text { Integ } \\
\text { Time } \\
\text { (sec) }\end{array}$ & $\begin{array}{c}r_{h} \\
(\mathrm{AU})\end{array}$ & $\begin{array}{c}\Delta \\
(\mathrm{AU})\end{array}$ & $\begin{array}{l}\text { Phase } \\
\text { Ang } \\
(0)\end{array}$ & $\begin{array}{c}\text { Tail }^{\mathrm{a}} \\
\text { Gas } \\
\text { PSAng } \\
(\circ)\end{array}$ & $\begin{array}{c}\text { Tail }^{\text {a }} \\
\text { Dust } \\
\text { PsAMV } \\
(\circ)\end{array}$ \\
\hline \multicolumn{10}{|l|}{ FOF176 } \\
\hline 06-04T03:35:31 & Imaging Dual & 19.71 & 29.5 & 616.0 & 1.708 & 1.688 & 34.76 & 105.75 & 78.33 \\
\hline 06-04T03:35:31 & Imaging Dual & 31.46 & 29.5 & 616.0 & & & & & \\
\hline 06-04T04:16:38 & Imaging Dual & 11.09 & 30.8 & 216.0 & & & & & \\
\hline 06-04T04:16:38 & Imaging Dual & 31.46 & 30.8 & 252.0 & & & & & \\
\hline \multicolumn{10}{|l|}{ FOF177 } \\
\hline 06-06Т04:15:14 & Imaging SWC & 19.71 & 45.0 & 198.0 & 1.684 & 1.711 & 34.76 & 104.16 & 77.47 \\
\hline 06-06Т04:29:07 & Grism LWC & $\mathrm{G} 227$ & 22.5 & 1800.0 & & & & & \\
\hline 06-06Т04:51:42 & Grism SWC & G111 & 24.0 & 1920.0 & & & & & \\
\hline \multicolumn{10}{|l|}{ FOF178 } \\
\hline 06-11T03:53:40 & Imaging SWC & 19.71 & 42.2 & 204.0 & 1.628 & 1.769 & 34.46 & 100.71 & 75.73 \\
\hline 06-11T03:57:33 & Grism LWC & G227 & 23.0 & 1748.0 & & & & & \\
\hline 06-11T04:32:22 & Grism SWC & G111 & 24.0 & 3336.0 & & & & & \\
\hline \multicolumn{10}{|l|}{ FOF179 } \\
\hline 06-13Т04:04:51 & Imaging SWC & 19.71 & 45.1 & 330.0 & 1.605 & 1.793 & 34.22 & 99.62 & 75.17 \\
\hline 06-13Т04:23:04 & Grism LWC & $\mathrm{G} 227$ & 23.0 & 1564.0 & & & & & \\
\hline 06-13Т04:46:21 & Grism SWC & G111 & 24.0 & 2016.0 & & & & & \\
\hline
\end{tabular}

*Notes. Orbital elements derived from JPL Horizons, ssd.jpl.nasa.gov/horizons.cgi.

${ }^{a}$ Vector direction measured CCW (eastward) from celestial north on the plane of the sky. 
Table 2. SOFIA Aperture Photometry and $\epsilon f \rho$ of Comet C/2012 K1 (PAN-STARRS)

\begin{tabular}{|c|c|c|c|c|c|}
\hline $\begin{array}{c}\text { Observation } \\
\text { Date } \\
\text { UT } 2014 \\
\text { (dd-mm hr:min:s) }\end{array}$ & $\begin{array}{l}\text { InstCfg } \\
\text { (Imaging) }\end{array}$ & $\begin{array}{c}\text { Fltr } \\
\lambda_{c} \\
(\mu \mathrm{m})\end{array}$ & $\begin{array}{c}\text { Flux } \\
\text { Density }^{\mathrm{a}} \\
(\mathrm{Jys})\end{array}$ & $\begin{array}{c}\lambda F_{\lambda} \\
\left(\times 10^{-16} \mathrm{~W} \mathrm{~cm}^{-2}\right)\end{array}$ & $\begin{array}{l}\epsilon f \rho \\
(\mathrm{cm})\end{array}$ \\
\hline \multicolumn{6}{|l|}{ FOF176 } \\
\hline 06-04T03:35:31 & DUAL & 19.71 & $15.102 \pm 1.117$ & $2.297 \pm 0.170$ & $14900 \pm 1100$ \\
\hline 06-04T03:35:31 & DUAL & 31.46 & $12.861 \pm 2.172$ & $1.226 \pm 0.207$ & $13000 \pm 2200$ \\
\hline 06-04T04:16:38 & DUAL & 11.09 & $6.119 \pm 1.217$ & $1.654 \pm 0.329$ & $16300 \pm 3200$ \\
\hline 06-04T04:16:38 & DUAL & 31.46 & $11.857 \pm 3.149$ & $1.130 \pm 0.300$ & $11900 \pm 3200$ \\
\hline \multicolumn{6}{|l|}{ FOF177 } \\
\hline 06-06Т04:15:14 & SWC & 19.71 & $18.338 \pm 2.343$ & $2.789 \pm 0.356$ & $17900 \pm 2300$ \\
\hline \multicolumn{6}{|l|}{ FOF178 } \\
\hline 06-11T03:53:40 & SWC & 19.71 & $16.962 \pm 2.246$ & $2.580 \pm 0.342$ & $16100 \pm 2100$ \\
\hline \multicolumn{6}{|l|}{ FOF179 } \\
\hline 06-13Т04:04:51 & SWC & 19.71 & $16.991 \pm 1.777$ & $2.584 \pm 0.270$ & $16000 \pm 1700$ \\
\hline
\end{tabular}

a Measured in a circular aperture with a radius of $9.984^{\prime \prime}$ centroided on the photocenter of the comet nucleus. 
Table 3. Best-fit Thermal Model Parameters and Derived Grain Mineralogy of Comet C/2012 K1 (Pan-STARRS)*

\begin{tabular}{lcc}
\hline \hline Dust component & $N_{p}{ }^{\mathrm{a}} \times 10^{16}$ & $\begin{array}{c}\text { Sub- } \mu \mathrm{m} \\
\text { mass fraction }\end{array}$ \\
\hline Amorphous pyroxene & $8526.433_{-1494.938}^{+1067.813}$ & $0.310_{-0.060}^{+0.043}$ \\
Amorphous olivine & $1228.326_{-640.688}^{+213.563}$ & $0.045_{-0.026}^{+0.009}$ \\
Amorphous carbon & $15246.560_{-427.125}^{+213.563}$ & $0.555_{-0.017}^{+0.009}$ \\
Crystalline olivine & $2476.880_{-854.250}^{+2135.626}$ & $0.090_{-0.031}^{+0.078}$ \\
Crystalline pyroxene & $0.000_{-0.000}^{+1067.813}$ & $0.000_{-0.000}^{+0.043}$ \\
\hline Other model parameters & & \\
\hline$\chi_{\nu}^{2}$ & 0.98 & \\
Degrees of freedom & $0.80_{-0.20}^{+0.25}$ & \\
Total submicron grain mass ${ }^{\mathrm{b}}\left(7.663_{-0.952}^{+1.310}\right) \times 10^{5} \mathrm{~kg}$ & \\
$f_{\text {cryst }}$ & $0.202_{-0.099}^{+0.297}$ & \\
\hline
\end{tabular}

*Notes. Uncertainties represent the $95 \%$ confidence level.

${ }^{a}$ Number of grains at the peak of the grain size distribution.

${ }^{\mathrm{b}}$ The total mass of the sub- $\mu \mathrm{m}$ sized grains contained within the spectral extraction aperture. 
Table 4. Thermal Modeling Dust Characteristics of Select Comets

\begin{tabular}{|c|c|c|c|c|c|c|}
\hline Comet Class & $\begin{array}{c}1 / a_{\text {orig }} \\
\left(10^{-6} \mathrm{AU}^{-1}\right)\end{array}$ & $\begin{array}{c}f_{\text {cryst }}{ }^{\mathrm{a}} \\
\text { Range } \\
(\%)\end{array}$ & $\begin{array}{l}\mathrm{SAC}^{\mathrm{b}} \\
\text { Ratio }\end{array}$ & $N$ & $\begin{array}{l}a_{\text {peak }} \\
(\mu \mathrm{m})\end{array}$ & Refs* \\
\hline \multicolumn{7}{|l|}{$\underline{\mathrm{NIC} / \mathrm{OC}^{\mathrm{c}}}$} \\
\hline C/2012 K1 (Pan-STARRS) & 42.9 & $10-50$ & $0.6-1.1$ & 3.4 & 0.6 & This work \\
\hline C/2007 N3 (Lulin) & 32.2 & $34-51$ & $0.42-0.54$ & 4.2 & 0.9 & {$[1]$} \\
\hline C/2001 Q4 (NEAT) & 61.2 & 71 & $1.7-5.7$ & 3.7 & 0.3 & {$[2,3]$} \\
\hline C/2002 V1 (NEAT) & 2279.3 & $66-69$ & 1.38 & 3.7 & 0.5 & {$[3]$} \\
\hline C/1995 O1 (Hale-Bopp) & 3805.0 & $60-78$ & $8.1-13.3$ & $3.4-3.7$ & 0.2 & {$[4,5]$} \\
\hline \multicolumn{7}{|l|}{$\mathrm{EC} / \mathrm{JFC}^{\mathrm{c}}$} \\
\hline 9P/Tempel 1 ( 1hrs post-impact, ctr $)$ & $\ldots$ & $19-25$ & $3.4-4.4$ & 3.7 & 0.2 & {$[7]$} \\
\hline 17P/Holmes & $\ldots$ & $\sim 42^{\mathrm{c}}$ & 0.2 & $\ldots$ & $\ldots$ & {$[7]$} \\
\hline 73P/SW3-B (Apert B) & $\ldots$ & $43-69$ & $1.09-1.59$ & 3.4 & 0.5 & {$[8]$} \\
\hline 73P/SW3-C (Apert M) & $\ldots$ & $57-69$ & $0.60-0.75$ & 3.4 & 0.3 & {$[8]$} \\
\hline
\end{tabular}

${ }^{a}$ Computed from thermal model sub- $\mu \mathrm{m}$ silicate mass fractions using Eqn.(1) described in $\$ 3.3$, where the range includes the model uncertainties when known.

${ }^{\mathrm{b}}$ Defined as the silicate-to-amorphous carbon ratio derived from thermal modelling of the SEDs where the range includes the model uncertainties when known.

${ }^{\mathrm{c}}$ Comet dynamical class divisions are $\mathrm{NIC} / \mathrm{OC}=$ Nearly Isotropic/Oort Cloud; EC/JFC = Ecliptic/Jupiter-Family

${ }^{\mathrm{d}}$ Estimated from Reach et al. (2010) who provide abundances weighted by grain surface area.

${ }^{*}$ References. (1) Woodward et al. (2011); (2) Wooden et al. (2004); (3) Ootsubo et al. (2007); (4) Harker et al. (2002); (5) Harker et al. (2004a); (6) Harker et al. (2007); (7) Reach et al. (2010); (8) Harker et al. (2011) 
Table 5. Af $\rho$ Values for Comet C/2012 K1 (PanSTARRS) on 2014 June 04.24 UT

\begin{tabular}{cccc}
\hline \hline $\begin{array}{c}\text { Aperture } \\
(\operatorname{arcsec})\end{array}$ & $\begin{array}{c}\rho \\
(\mathrm{km})\end{array}$ & $\begin{array}{c}R \\
(\mathrm{mag})\end{array}$ & $\begin{array}{c}\text { Af } \rho \\
(\mathrm{cm})\end{array}$ \\
\hline 11.24 & 6705 & $12.157 \pm 0.008$ & $5731 \pm 42$ \\
19.97 & 11912 & $11.593 \pm 0.009$ & $5424 \pm 43$ \\
25.14 & 14999 & $11.344 \pm 0.009$ & $5417 \pm 43$ \\
33.53 & 19999 & $11.065 \pm 0.009$ & $5253 \pm 41$ \\
41.91 & 24999 & $10.835 \pm 0.009$ & $5194 \pm 41$ \\
50.29 & 29999 & $10.674 \pm 0.009$ & $5020 \pm 39$ \\
\hline
\end{tabular}

${ }^{a}$ Effective circular aperture diameter.

${ }^{\mathrm{b}} \mathrm{Af} \rho$ values corrected to zero phase (see $\oint_{3.5}$ ). 